\title{
NORI FUNDAMENTAL GERBE OF ESSENTIALLY FINITE COVERS AND GALOIS CLOSURE OF TOWERS OF TORSORS
}

\author{
MARCO ANTEI, INDRANIL BISWAS, MICHEL EMSALEM, FABIO TONINI, AND LEI ZHANG
}

\begin{abstract}
We prove the existence of a Galois closure for towers of torsors under finite group schemes over a proper, geometrically connected and geometrically reduced algebraic stack $X$ over a field $k$. This is done by describing the Nori fundamental gerbe of an essentially finite cover of $X$. A similar result is also obtained for the S-fundamental gerbe.
\end{abstract}

\section{INTRODUCTION}

Let $K \subseteq L$ be a finite separable field extension. The extension is called Galois if all automorphisms of the algebraic closure $\bar{L}$ fixing $K$ send $L$ to $L$. In a more geometric language this means that $K \subseteq L$ is Galois if and only if $\operatorname{Spec} L \longrightarrow \operatorname{Spec} K$ is a torsor under the automorphism group $\operatorname{Aut}_{K}(L)$. It is not true that all finite separable extensions are Galois. Even worse, it could happen that even for two successive Galois extensions $K \subseteq E$ and $E \subseteq L$ the tower $K \subseteq L$ is, although still finite separable, not Galois anymore. For example, $\mathbb{Q} \subseteq \mathbb{Q}[\sqrt{2}] \subseteq \mathbb{Q}[\sqrt[4]{2}]$ is a tower of Galois extensions, but itself is not Galois. Since $L=K[\beta]$ for some $\beta \in L$, there is a field $L^{\prime}$ inside a chosen algebraic closure $\bar{L}$ of $L$, obtained from $L$ by adjoining all the roots of the minimal polynomial of $\beta$ to $L$. The extension $K \subseteq L^{\prime}$ enjoys the following properties:

- The extension $K \subseteq L^{\prime}$ is Galois.

- For any Galois extension $K \subseteq M$ inside $\bar{L}$ with $M$ containing $L$, we have $L^{\prime} \subseteq M$. The field $L^{\prime}$, or more precisely the extension $K \subseteq L^{\prime}$, is called the Galois closure of $K \subseteq L$. When $K \subseteq L$ is a tower of two successive Galois extensions $K \subseteq E$ and $E \subseteq L$, then $L \subseteq L^{\prime}, E \subseteq L^{\prime}$ are also Galois extensions. If we denote $G:=\operatorname{Aut}_{K}(E), H:=\operatorname{Aut}_{E}(L)$, then the Galois closure $L^{\prime}$ provides the following data $(\diamond)$ :

- The automorphism group $\mathcal{G}:=\operatorname{Aut}_{K}\left(L^{\prime}\right)$ and group homomorphisms $\alpha: \mathcal{G} \rightarrow G$ and $\operatorname{Ker}(\alpha)=\operatorname{Aut}_{E}\left(L^{\prime}\right) \rightarrow H$.

- A $\mathcal{G}$-torsor, namely Spec $L^{\prime} \rightarrow \operatorname{Spec} K$, together with a factorization $\operatorname{Spec} L^{\prime} \longrightarrow$ $\operatorname{Spec} L$ such that $\operatorname{Spec} L^{\prime} \rightarrow \operatorname{Spec} E$ is $\mathcal{G}$-equivariant and $\operatorname{Spec} L^{\prime} \longrightarrow \operatorname{Spec} L$ is $\operatorname{Ker}(\alpha)$-equivariant.

Date: November 20, 2018.

2010 Mathematics Subject Classification. 14F35, $14 \mathrm{D} 23$.

Key words and phrases. Nori fundamental gerbe, essentially finite bundle, essentially finite cover, algebraic stack.

This work is supported by the departmental fund of the Chinese University of Hong Kong and the Labex CEMPI (ANR-11-LABX-01). The second author is supported by the J. C. Bose Fellowship. 
In the construction of the étale fundamental group, Grothendieck completely generalized the Galois theory of fields to that of schemes. In the world of schemes a "finite separable field extension" becomes a finite étale morphism, and a "Galois extension" becomes a connected finite étale cover which is a torsor under its automorphism group, namely a Galois cover. Let $X$ be a connected scheme equipped with a geometric point $x: \operatorname{Spec} \bar{k} \longrightarrow X$, and let $f: Z \longrightarrow X$ be a connected surjective finite étale cover. Using Grothendieck's equivalence between the category of finite étale covers of $X$ and the category of finite sets with a continuous $\pi_{1}^{\text {et }}(X, x)$-action, one can identify $f$ with a finite set with a transitive $\pi_{1}^{\text {ét }}(X, x)$-action or, after a choice of a geometric point $z$ in $Z$, an open subgroup $\pi$ of $\pi_{1}^{\text {ét }}(X, x)$. Thus there is a pointed finite étale cover $f^{\prime}: Z^{\prime} \longrightarrow X$ mapping to $Z \longrightarrow X$ given by the maximal normal subgroup of $\pi_{1}^{\text {et }}(X, x)$ contained in $\pi$, and this pointed cover enjoys the following properties $(\neg)$ :

- The cover $f^{\prime}: Z^{\prime} \longrightarrow X$ is Galois.

- The pointed cover $\left(Z^{\prime}, z^{\prime}\right)$ with the map $\left(Z^{\prime}, z^{\prime}\right) \longrightarrow(Z, z)$ has the following universal property: if $(T, t) \longrightarrow(X, x)$ is another pointed Galois cover mapping to $(Z, z)$, then there exists a unique map $(T, t) \longrightarrow\left(Z^{\prime}, z^{\prime}\right)$ factorising $\left(Z^{\prime}, z^{\prime}\right) \longrightarrow(Z, z)$.

Note that since $f^{\prime}$ corresponds to a normal subgroup of $\pi_{1}^{\text {ét }}(X, x)$ it is independent of the choice of the base points $z, x$. The map $f^{\prime}$ is the Galois closure of $f$ (see also [Sz, Proposition 5.3.9, pp. 169] for a different approach). If $f$ is moreover a tower of Galois covers $Z \longrightarrow Y$ and $Y \longrightarrow X$, then replacing the automorphisms of field extensions by the automorphisms of covers the Galois closure $Z^{\prime} \longrightarrow X$ enjoys exactly the same properties $(\diamond)$ listed in the field case.

Now a natural question is: what about covers which may not be étale such as the Kummer covers in characteristic $p>0$ ? What would be a "Galois closure" in that case? The present paper is set out to address these issues. As in the étale case we resort to the machinery of fundamental groups. Here the fundamental group is the Nori fundamental group $\pi^{\mathrm{N}}(-, *)$ which was introduced in [No1] or its generalization - the Nori fundamental gerbe $\Pi_{-}^{\mathrm{N}}$ introduced in [BV]. In Definition 1.1 we define the notion of the Nori fundamental gerbe. Its existence is characterized in 1.2, and its Tannakian property is in Theorem 1.10. We assume the base $\mathcal{X}$ to be inflexible to guarantee the existence of the fundamental gerbe, and this "inflexible" property plays the same role as the "connected" property in the étale case. We assume $\mathcal{X}$ to be pseudo-proper (Definition 1.6) in order to use the Tannakian property. The next step is to understand what are the "finite separable extensions" or the "finite étale morphisms" in this context. For this, we introduce the notion of "essentially finite covers" in Definition 1.11. The "Galois extensions" or the "Galois covers" are replaced by "Nori-reduced torsors" (Definition 1.13). Under this setting we obtain a very pretty Galois theory:

Theorem I. Let $\mathcal{X}$ be a pseudo-proper and inflexible algebraic stack of finite type over $k$ and let $f: \mathcal{Y} \longrightarrow \mathcal{X}$ be an essentially finite cover. If char $k>0$ assume that either $f$ is étale or $\operatorname{dim}_{k} \mathrm{H}^{1}(\mathcal{X}, E)<\infty$ for all vector bundles $E$ on $\mathcal{X}$. Then 
(1) There exists a finite map $\Pi \longrightarrow \Pi_{\mathcal{X} / k}^{\mathrm{N}}$, which is unique up to equivalence, whose base change along $\mathcal{X} \longrightarrow \Pi_{\mathcal{X} / k}^{\mathrm{N}}$ is $\mathcal{Y} \stackrel{f}{\longrightarrow} \mathcal{X}$. Moreover, $\mathcal{Y}$ is inflexible over $k$ if and only if $\Pi$ is a gerbe over $k$. In this case $\mathcal{Y} \longrightarrow \Pi$ is the Nori fundamental gerbe of $\mathcal{Y}$, and there is a 2-Cartesian diagram,

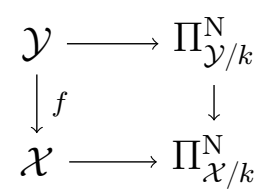

and we have $\operatorname{EFin}(\operatorname{Vect}(\mathcal{Y}))=\left\{V \in \operatorname{Vect}(\mathcal{Y}) \mid f_{*} V \in \operatorname{EFin}(\operatorname{Vect}(\mathcal{X}))\right\}$, where $\operatorname{EFin}(\operatorname{Vect}(\mathcal{X}))$ denotes the category of essentially finite vector bundles on $\mathcal{X}$ (see Definition 1.5).

(2) The étale case. If $f$ is étale then $\mathcal{Y}$ is inflexible over $k$ if and only if $\mathrm{H}^{0}\left(\mathcal{Y}, \mathcal{O}_{\mathcal{Y}}\right)=k$ and in this case there are 2-Cartesian diagrams

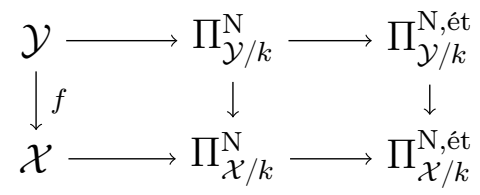

(3) The torsor case. If $f$ is a torsor under a finite group scheme $G$ over $k$ the following are equivalent:

- $\mathcal{Y}$ is inflexible over $k$;

- $\mathrm{H}^{0}\left(\mathcal{Y}, \mathcal{O}_{\mathcal{Y}}\right)=k$;

- $f$ is Nori-reduced over $k$.

Under these conditions there are 2-Cartesian diagrams:

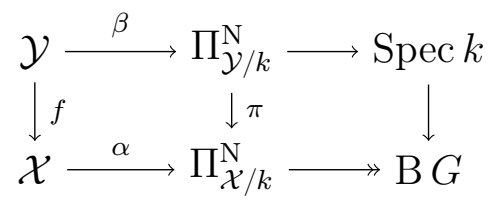

For pointed covers Theorem I yields the following Galois correspondence.

Corollary I. Let $\mathcal{X}$ be a pseudo-proper and inflexible algebraic stack of finite type over $k$ with a rational point $x \in \mathcal{X}(k)$. If char $k>0$ assume that $\operatorname{dim}_{k} \mathrm{H}^{1}(\mathcal{X}, E)<\infty$ for all vector bundles $E$ on $\mathcal{X}$. Then there is an equivalence of categories

$$
\left\{\begin{array}{c}
\text { Pointed essentially finite covers } \\
(\mathcal{Y}, y) \longrightarrow(\mathcal{X}, x) \text { with } \mathcal{Y} \text { inflexible }
\end{array}\right\} \longrightarrow\left\{\begin{array}{c}
\text { Subgroups } H<\pi^{\mathrm{N}}(\mathcal{X}, x) \\
\text { of finite index }
\end{array}\right\}
$$

where in the right-hand side we consider inclusions as arrows. Moreover 
- an essentially finite cover $(\mathcal{Y}, y) \longrightarrow(\mathcal{X}, x)$ with $\mathcal{Y}$ inflexible is a torsor under a finite group $G$ if and only if $\pi^{\mathrm{N}}(\mathcal{Y}, y)$ is a normal subgroup of $\pi^{\mathrm{N}}(\mathcal{X}, x)$ and in this case there is an exact sequence

$$
1 \longrightarrow \pi^{\mathrm{N}}(\mathcal{Y}, y) \longrightarrow \pi^{\mathrm{N}}(\mathcal{X}, x) \longrightarrow G \longrightarrow 1
$$

- an essentially finite cover $(\mathcal{Y}, y) \longrightarrow(\mathcal{X}, x)$ with $\mathcal{Y}$ inflexible is étale if and only if the finite scheme $\pi^{\mathrm{N}}(\mathcal{X}, x) / \pi^{\mathrm{N}}(\mathcal{Y}, y)$ is étale over $k$.

The above sequence was already proved to be exact in [EHS, Theorem 2.9] under the assumption that $G$ is étale. The main difficulty in the present work is to show that, if $f: \mathcal{Y} \longrightarrow \mathcal{X}$ is an essentially finite cover, then $f_{*}$ preserves essentially finite vector bundles. The statement would be false without assuming that $\mathcal{X}$ is pseudo-proper and $\operatorname{dim}_{k} \mathrm{H}^{1}(\mathcal{X}, E)<\infty$ (see Lemmas 2.11, 2.13 and, for a counter-example, Example 5.5). A key tool in the proof is a characterization of essentially finite vector bundles given in [TZ2] which generalizes previous results of [BdS] and [AM].

Here is our main result on finding Galois closures for covers which may not be étale:

Theorem II. Let $\mathcal{X}$ be a pseudo-proper and inflexible fibered category over $k$ and $f: \mathcal{Y} \longrightarrow$ $\mathcal{X}$ an essentially finite cover with a rational point $y \in \mathcal{Y}(k)$. Denote by $\Gamma$ the monodromy gerbe of $f_{*} \mathcal{O}_{\mathcal{Y}}$ in $\operatorname{EFin}(\operatorname{Vect}(\mathcal{X}))$ and by $\Delta \longrightarrow \Gamma$ the cover in (1) of Lemma 2.2 that extends $f$. Then

(1) There is a diagram with Cartesian squares

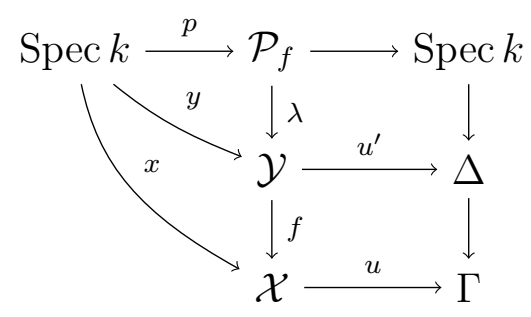

such that the map $\pi: \mathcal{P}_{f} \longrightarrow \mathcal{X}$ is a pointed Nori-reduced torsor under the finite group scheme $G_{f}=\underline{\operatorname{Aut}}_{\Gamma}(u(x))$, and $\Gamma$ is the monodromy gerbe of $\pi_{*} \mathcal{O}_{\mathcal{P}_{f}}$.

(2) The map $\lambda: \mathcal{P}_{f} \longrightarrow \mathcal{Y}$ is faithfully flat if and only if $\Delta$ is a gerbe (e.g. when $\mathcal{Y}$ is inflexible) in which case it is a Nori-reduced torsor for a subgroup scheme of $G_{f}$.

(3) In the general case $\lambda: \mathcal{P}_{f} \longrightarrow \mathcal{Y}$ factors as $\eta \circ \lambda^{\prime}$, where $\lambda^{\prime}: \mathcal{P}_{f} \longrightarrow \mathcal{Y}^{\prime}$ is faithfully flat and a Nori-reduced torsor for a finite subgroup scheme of $G_{f}$, and $\eta: \mathcal{Y}^{\prime} \longrightarrow \mathcal{Y}$ is a closed immersion.

(4) Finally the torsor $\pi: \mathcal{P}_{f} \longrightarrow \mathcal{X}$ has the following universal property: for any pointed Nori-reduced torsor $g:(\mathcal{T}, t) \longrightarrow(\mathcal{X}, x)$ for a finite group scheme $G$, and any pointed faithfully flat $\mathcal{X}$-morphism $h:(\mathcal{T}, t) \rightarrow(\mathcal{Y}, y)$, there is a unique factorization $h=\lambda \circ j$, where $j:(\mathcal{T}, t) \longrightarrow\left(\mathcal{P}_{f}, p\right)$ is equivariant with respect to a surjective homomorphism $G \longrightarrow G_{f}$. 
We then define the Galois closure of a pointed essentially finite cover $f: \mathcal{Y} \longrightarrow \mathcal{X}$ to be the Nori-reduced torsor $\mathcal{P}_{f} \longrightarrow \mathcal{X}$ in Theorem II. In particular, if $f: \mathcal{Y} \longrightarrow \mathcal{X}$ is a pointed inflexible essentially finite cover (see Definition 2.4), then it admits a Galois closure in the sense of (汿) (see Lemma 2.6).

Next we deal with the Galois closure problem for towers. Let $G$ and $H$ be finite group schemes over a field $k$. A $(G, H)$-tower of torsors over a $k$-algebraic stack $\mathcal{X}$ consists of maps $f: \mathcal{Z} \stackrel{h}{\longrightarrow} \mathcal{Y} \stackrel{g}{\longrightarrow} \mathcal{X}$ where $h$ and $g$ are an $H$-torsor and a $G$-torsor respectively. We first show that under some conditions $f=g \circ h$ is an essentially finite cover (Lemma 3.4). Then we define the notion of a Galois envelope (see Definition 3.8) in the sense of $(\diamond)$. We investigate when the torsor $\mathcal{P}_{f}$ obtained in Theorem II for $\mathcal{Z} \longrightarrow \mathcal{X}$ is a Galois envelope, if $\mathcal{P}_{f}$ is really a Galois envelope then we call it the Galois closure for the tower $\mathcal{Z} \stackrel{h}{\longrightarrow} \mathcal{Y} \stackrel{g}{\longrightarrow} \mathcal{X}$. Here we do not insist that $g, h$ are Nori-reduced torsors (which are "Galois covers" in the étale case). In order to obtain the Galois envelope we study the moduli $\mathrm{B}(G, H)$ of all $(G, H)$-towers (Definition 3.1) which is an algebraic stack locally of finite type over $k$ (Proposition 3.3). Our main result for pointed towers is the following:

Theorem III. Let $\mathcal{X}$ be a pseudo-proper (see Definition 1.6) and inflexible (see Definition 1.1) algebraic stack of finite type over $k$. If char $k>0$ assume that $\operatorname{dim}_{k} \mathrm{H}^{1}(\mathcal{X}, E)<\infty$ for all vector bundles $E$ on $\mathcal{X}$. Then, if $\mathcal{Z} \stackrel{h}{\longrightarrow} \mathcal{Y} \stackrel{g}{\longrightarrow} \mathcal{X}$ is a $(G, H)$-tower of torsors then $\mathcal{Z} \longrightarrow \mathcal{X}$ is an essentially finite cover. Assume moreover that $\mathcal{Z}$ has a $k$-point $z \in \mathcal{Z}(k)$. Then the tower admits a pointed Nori-reduced Galois closure $(\mathcal{P}, p) \stackrel{\lambda}{\longrightarrow}(\mathcal{Z}, z)$ such that:

(1) $\lambda$ is faithfully flat if and only if the torsors in the tower are Nori-reduced, or equivalently $\mathcal{Z}$ and $\mathcal{Y}$ are inflexible, and in this case it is a Nori-reduced torsor under a finite group scheme.

(2) $\mathcal{P} \longrightarrow \mathcal{X}$ is a torsor under a finite subgroup of the affine and of finite type $k$-group scheme $\underline{\operatorname{Aut}}_{\mathrm{B}(G, H)}(\xi)$, where $\xi \in \mathrm{B}(G, H)(k)$ is the tower fiber of the given tower over $x=g h(z):$ Spec $k \longrightarrow \mathcal{X}$.

Previous attempts to give an affirmative answer to this question failed: both [Ga] and [ABE] (unpublished) contain mistakes in the proof of their main theorems. In [Ga], Garuti claims a functorial construction of the "Galois closure" (which is the Galois envelope in our paper) for all towers of torsors under finite locally free group schemes over any locally Noetherian base $B$. He works basically without any assumption. But this is too good to be true. Without rational points the claim is wrong, otherwise the map in [Zh, Corollary 5.14, pp. 41] would be an isomorphism. Example 5.4 shows that even for a smooth connected affine scheme $X$ over an algebraically closed field $k$ there are pointed towers without a Galois envelope (because $X$ is not pseudo-proper). Example 5.3 shows that the assumption $\operatorname{dim}_{k} \mathrm{H}^{1}(\mathcal{X}, E)<\infty$ is also important. This condition is needed to ensure that every $\mathbb{G}_{a}$-torsor over a cover of $\mathcal{X}$ is induced by a torsor under a finite subgroup of $\mathbb{G}_{a}$. Notice that the cohomological assumption is met for proper algebraic stacks of finite type over $k$ ([Fal]). Moreover a geometrically connected and geometrically reduced algebraic stack over $k$ is inflexible (see Remark 1.2). 
Recently, in [Otabe] S. Otabe used our result to study the lifting problem for linearly reductive torsors over curves. He also studied the Galois closure problem in the appendix for non-proper base schemes.

We also study a similar problem for the $S$-fundamental gerbe (see [BPS], [BHD], [La1], [La2]). Let $\operatorname{Ns}(\mathcal{X})$ be the category of semi-stable vector bundles on $\mathcal{X}$ (see Definition 4.1). The following analog of Theorem I is proved for $S$-fundamental gerbes.

Theorem IV. Let $\mathcal{X}$ be a pseudo-proper algebraic stack of finite type over $k$ and with an $\mathrm{S}$-fundamental gerbe. Then $\mathcal{X}$ is inflexible and the profinite quotient of the S-fundamental gerbe of $\mathcal{X}$ over $k$ is the Nori fundamental gerbe of $\mathcal{X}$ over $k$. Let also $f: \mathcal{Y} \longrightarrow \mathcal{X}$ be an essentially finite cover with $\mathcal{Y}$ inflexible and, if char $k>0$, assume that $\operatorname{dim}_{k} \mathrm{H}^{1}(\mathcal{X}, E)<\infty$ for all vector bundles $E$ on $\mathcal{X}$. Then

$$
\operatorname{Ns}(\mathcal{Y})=\left\{V \in \operatorname{Vect}(\mathcal{Y}) \mid f_{*} V \in \operatorname{Ns}(\mathcal{X})\right\}
$$

and $\mathcal{Y}$ has an $\mathrm{S}$-fundamental gerbe fitting in a 2-Cartesian diagram

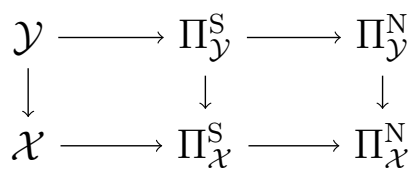

In particular if $y \in \mathcal{Y}(k)$ and $x=f(y) \in \mathcal{X}(k)$ then there is a Cartesian diagram of affine group schemes

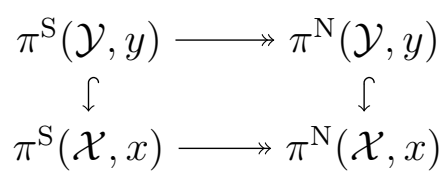

When $f: \mathcal{Y} \longrightarrow \mathcal{X}$ is a pointed torsor under a finite group scheme $G$, then the following sequence is exact

$$
1 \longrightarrow \pi^{\mathrm{S}}(\mathcal{Y}, y) \longrightarrow \pi^{\mathrm{S}}(\mathcal{X}, x) \longrightarrow G \longrightarrow 1
$$

The paper is divided as follows. In the first section we recall part of the machinery about Nori fundamental gerbes and prove some preliminary results. In the second section we study essentially finite covers and their Nori fundamental gerbes, proving in particular Theorem I, Theorem II and Corollary I. In the third section we study towers of torsors and their Galois closures, proving Theorems 3.9 and III, while in the fourth section we study the $S$-fundamental gerbe and prove Theorem IV. Finally in the last section we collect some counter-examples.

\section{ACKNOWLEDGEMENT}

We would like to thank Hélène Esnault and Angelo Vistoli for helpful conversations and suggestions received. We would also like to thank the referee for many helpful remarks. 


\section{Notation And Preliminaries}

1.1. Notation. By a fibered category over a scheme $S$ we will always mean a category fibered in groupoids over the category Aff/S of affine schemes over $S$.

Let $\mathcal{X}$ be a fibered category over Aff $/ S$, where $S$ is an affine scheme. An fpqc-altlas from a scheme $U$ is a map $U \longrightarrow \mathcal{X}$ representable by fpqc-coverings of algebraic spaces, i.e. for any algebraic space $T$ mapping to $\mathcal{X}$ the fibered product $U \times{ }_{\mathcal{X}} T$ is again an algebraic space over $S$ which is an fpqc covering of $T$. A fibered category $\mathcal{X}$ is said to be quasi-compact if it has an fpqc-atlas from a quasi-compact scheme. If $f: \mathcal{X} \longrightarrow \mathcal{Y}$ is a quasi-compact and quasi-separated map between fibered categories admitting fpqc-atlases from schemes, then the pullback $f^{*}: \mathrm{QCoh}(\mathcal{Y}) \longrightarrow \mathrm{QCoh}(\mathcal{X})$ admits a right adjoint $f_{*}: \mathrm{QCoh}(\mathcal{X}) \longrightarrow \mathrm{QCoh}(\mathcal{Y})$ which commutes with flat base change, where $\mathrm{QCoh}(-)$ denotes the category of quasi-coherent sheaves (see [Ton, Proposition 1.7]).

A cover of a fibered category $\mathcal{X}$ is a finite, flat and finitely presented morphism or, equivalently, an affine map $f: \mathcal{Y} \longrightarrow \mathcal{X}$ with the property that $f_{*} \mathcal{O}_{\mathcal{Y}}$ is locally free of finite rank. If $\mathcal{X}$ is defined over a field $k$ a pointed cover over $k$

$$
(\mathcal{Y}, y) \longrightarrow(\mathcal{X}, x)
$$

is a cover $f: \mathcal{Y} \longrightarrow \mathcal{X}$ with $x \in \mathcal{X}(k)$ and $y \in \mathcal{Y}_{x}(k)$, where $\mathcal{Y}_{x}$ denotes the fiber of $f$ over $x$, which is a finite $k$-scheme; equivalently $y \in \mathcal{Y}(k)$ with a given isomorphism $f(y) \simeq x$.

Given a morphism of schemes $U \longrightarrow V$ and a functor $F:$ Aff $/ U \longrightarrow$ (Sets), the Weil restriction of $F$ along $U \longrightarrow V$ is the functor

$$
W_{U / V}(F): \text { Aff } / V \longrightarrow(\operatorname{Sets}), Z \longmapsto \operatorname{Hom}\left(Z \times_{V} U, F\right) .
$$

Given any functor $G:$ Aff $/ V \longrightarrow\left(\right.$ Sets), we set $W_{U}(G):=W_{U / V}\left(G \times{ }_{V} U\right)$.

Injectivity and surjectivity of morphisms of group schemes always mean the corresponding properties for fpqc sheaves. For affine group schemes over a field an injective morphism is a closed immersion and a surjective morphism is faithfully flat ([Wat, Theorem 15.5] for surjectivity).

1.2. Preliminaries. We will now recall some results used in later sections.

Fix a base field $k$.

For properties of affine gerbes over a field (often improperly called just gerbes) and Tannakian categories used here the reader is referred to [TZ1, Appendix B].

Definition 1.1 ([BV, Definition 5.1, Definition 5.3]). For a fibered category $\mathcal{X}$ over $k$, the Nori fundamental gerbe (respectively, Nori étale fundamental gerbe) of $\mathcal{X} / k$ is a profinite (respectively, proétale) gerbe $\Pi$ over $k$ together with a map $\mathcal{X} \longrightarrow \Pi$ such that for all finite (respectively, finite and étale) stacks $\Gamma$ over $k$ the pullback functor

$$
\operatorname{Hom}_{k}(\Pi, \Gamma) \longrightarrow \operatorname{Hom}_{k}(\mathcal{X}, \Gamma)
$$

is an equivalence of categories. Furthermore, if this gerbe exists, it is unique up to a unique isomorphism and in that case it will be denoted by $\Pi_{\mathcal{X} / k}^{\mathrm{N}}\left(\right.$ respectively, $\left.\Pi_{\mathcal{X} / k}^{\mathrm{N} \text {,ét }}\right)$; sometimes $/ k$ will be dropped if it is clear from the context. 
We call $\mathcal{X}$ inflexible if it is non-empty and all maps from it to a finite stack over $k$ factor through a finite gerbe over $k$.

Remark 1.2. By [BV, p. 13, Theorem 5.7] $\mathcal{X}$ admits a Nori fundamental gerbe if and only if it is inflexible; in this case, the Nori étale fundamental gerbe of $\mathcal{X}$ is the maximal proétale quotient of the Nori fundamental gerbe of $\mathcal{X}$. If $\mathcal{X}$ is reduced, quasi-compact and quasi-separated, then $\mathcal{X}$ is inflexible if and only if $k$ is algebraically closed in $\mathrm{H}^{0}\left(\mathcal{O}_{\mathcal{X}}\right)$ [TZ1, Theorem 4.4]. In particular if $\mathcal{X}$ is geometrically connected and geometrically reduced, then it is inflexible.

Definition 1.3. If $\mathcal{X}$ is an inflexible fibered category over $k$ with a rational point $x \in \mathcal{X}(k)$ and Nori gerbe $\psi: \mathcal{X} \longrightarrow \Pi_{\mathcal{X} / k}^{\mathrm{N}}$, the Nori fundamental group scheme $\pi^{\mathrm{N}}(\mathcal{X} / k, x)$ of $(\mathcal{X}, x)$ over $k$ is the sheaf of automorphisms of $\psi(x) \in \Pi_{\mathcal{X} / k}^{\mathrm{N}}(k)$. Again $/ k$ will often be dropped if it is clear from the context.

Remark 1.4. The Nori fundamental group scheme $\pi^{\mathrm{N}}(\mathcal{X}, x)$ is a profinite group scheme and its classifying stack $\mathrm{B} \pi^{\mathrm{N}}(\mathcal{X}, x)$ is isomorphic to $\Pi_{\mathcal{X}}^{\mathrm{N}}$ (the trivial torsor is sent to $\psi(x)$ ). The universal property of $\Pi_{\mathcal{X}}^{\mathrm{N}}$ translates into the following: for all finite group schemes $G$ over $k$ the map

$$
\begin{gathered}
\operatorname{Hom}_{k \text {-groups }}\left(\pi^{\mathrm{N}}(\mathcal{X}, x), G\right) \longrightarrow\{\text { pointed } G \text {-torsors }(\mathcal{P}, p) \longrightarrow(\mathcal{X}, x)\} / \simeq \\
\left(\pi^{\mathrm{N}}(\mathcal{X}, x) \longrightarrow G\right) \longmapsto\left(\mathcal{X} \longrightarrow \mathrm{B} \pi^{\mathrm{N}}(\mathcal{X}, x) \longrightarrow \mathrm{B} G\right)
\end{gathered}
$$

is bijective.

Definition 1.5 ([BV, p. 21, Definition 7.7]). Let $\mathcal{C}$ be an additive and monoidal category. An object $E \in \mathcal{C}$ is called finite if there exist polynomials $f \neq g \in \mathbb{N}[X]$ and an isomorphism $f(E) \simeq g(E)$; the object $E$ is called essentially finite if it is a kernel of a homomorphism of finite objects of $\mathcal{C}$. Let $\operatorname{EFin}(\mathcal{C})$ denote the full subcategory of $\mathcal{C}$ consisting of essentially finite objects.

Definition 1.6 ([BV, p. 20, Definition 7.1]). A category $\mathcal{X}$ fibered in groupoids over a field $k$ is pseudo-proper if it satisfies the following two conditions:

(1) there exists a quasi-compact scheme $U$ together with a morphism $U \longrightarrow \mathcal{X}$ which is representable, faithfully flat, quasi-compact, and quasi-separated, and

(2) for all vector bundles $E$ on $\mathcal{X}$ the $k$-vector space $\mathrm{H}^{0}(\mathcal{X}, E)$ is finite-dimensional.

Example 1.7 ([BV, p. 20, Example 7.2]). Examples of pseudo-proper fibered categories are proper algebraic stacks and affine gerbes.

Remark 1.8. Let $\mathcal{X}$ be a pseudo-proper algebraic stack of finite type over $k$. If $\mathcal{X}$ is inflexible then $\mathrm{H}^{0}\left(\mathcal{O}_{\mathcal{X}}\right)=k$ (see [BV, Lemma 7.4]), while the converse holds if $\mathcal{X}$ is reduced (see Remark 1.2).

Definition 1.9. Let $\mathcal{C}$ be a Tannakian category, and let $M \in \mathcal{C}$ be an object. The monodromy gerbe of $M$ is the gerbe corresponding to the Tannakian subcategory of $\mathcal{C}$ generated by $M$. (See [TZ1, Definition B.8, pp. 42]) 
Theorem 1.10 ([BV, p. 22, Theorem 7.9, Corollary 7.10]). Let $\mathcal{X}$ be an inflexible pseudoproper fibered category over a field $k$. Then the pullback along $\mathcal{X} \longrightarrow \Pi_{\mathcal{X} / k}^{\mathrm{N}}$ induces an equivalence of categories $\operatorname{Vect}\left(\Pi_{\mathcal{X} / k}^{\mathrm{N}}\right) \longrightarrow \operatorname{EFin}(\operatorname{Vect}(\mathcal{X}))$.

Let $\mathcal{C}$ be a Tannakian category. Then $\operatorname{EFin}(\mathcal{C})$ is the Tannakian subcategory of $\mathcal{C}$ of objects whose monodromy gerbe is finite.

Definition 1.11. A cover $f: \mathcal{Y} \longrightarrow \mathcal{X}$ is essentially finite if $f_{*} \mathcal{O}_{\mathcal{Y}}$ is an essentially finite vector bundle.

Definition 1.12. Let $\mathcal{X}$ be an inflexible and pseudo-proper fibered category over a field $k$. Given an object $V$ of $\operatorname{EFin}(\operatorname{Vect}(\mathcal{X}))$, the gerbe corresponding to the full Tannakian subcategory of EFin $(\operatorname{Vect}(\mathcal{X}))$ generated by $V$ will be called the monodromy gerbe of $V$. When $f: \mathcal{Y} \longrightarrow \mathcal{X}$ is an essentially finite cover, the monodromy gerbe of the cover is by definition the monodromy gerbe of $f_{*} \mathcal{O}_{\mathcal{Y}}$.

Definition 1.13 ([BV, Definition 5.10]). A map $\mathcal{X} \longrightarrow \Gamma$ from a fibered category over $k$ to a finite gerbe over $k$ is called Nori-reduced over $k$ if any faithful morphism $\Gamma^{\prime} \stackrel{\alpha}{\longrightarrow} \Gamma$ that fits in a factorization $\mathcal{X} \longrightarrow \Gamma^{\prime} \stackrel{\alpha}{\longrightarrow} \Gamma$, where $\Gamma^{\prime}$ is a gerbe, is an isomorphism.

A torsor $\mathcal{P} \longrightarrow \mathcal{X}$ under a finite group scheme $G$ over $k$ is called Nori-reduced over $k$ if the map $\mathcal{X} \longrightarrow \mathrm{B} G$ is Nori-reduced over $k$.

Remark 1.14. If $\mathcal{X}$ is inflexible, then any map from $\mathcal{X}$ to a finite gerbe factors uniquely through a Nori-reduced map (see [BV, Lemma 5.12]). Moreover $\Pi_{\mathcal{X}}^{\mathrm{N}}$ can be seen as the projective limit of the Nori-reduced maps $\mathcal{X} \longrightarrow \Gamma$ (see [BV, Theorem 5.7] and its proof).

If $\mathcal{X}$ is an inflexible and pseudo-proper fibered category, and $\phi: \mathcal{X} \longrightarrow \Gamma$ is a map to a finite gerbe, then $\phi$ is Nori-reduced if and only if the induced map $\Pi_{\mathcal{X}}^{\mathrm{N}} \longrightarrow \Gamma$ is a quotient ([TZ1, Definition B.1, pp. 40]); in this case $\operatorname{Vect}(\Gamma) \longrightarrow \operatorname{EFin}(\operatorname{Vect}(\mathcal{X})$ ) is a Tannakian subcategory. This is a direct consequence of Theorem 1.10 and the universal property of $\Pi_{\mathcal{X}}^{\mathrm{N}}$. Moreover $\phi_{*} \mathcal{O}_{\mathcal{X}} \simeq \mathcal{O}_{\Gamma}($ see $[\mathrm{BV}$, Lemma 7.11]).

One of the key ingredients in the paper is the following result.

Theorem 1.15 ([TZ2, Corollary I]). Let $\mathcal{X}$ be a pseudo-proper and inflexible algebraic stack of finite type over a field $k$ of positive characteristic, and let $f: \mathcal{Y} \longrightarrow \mathcal{X}$ be a surjective cover. If $V \in \operatorname{Vect}(\mathcal{X})$, and $f^{*} V$ is free, then $V$ is essentially finite in $\operatorname{Vect}(\mathcal{X})$.

Remark 1.16. If $\mathcal{X}$ is a pseudo-proper and inflexible algebraic stack of finite type over a field $k$ (the characteristic is allowed to be 0 ), $f: \mathcal{Y} \longrightarrow \mathcal{X}$ is a surjective étale cover and $V \in \operatorname{Vect}(\mathcal{X})$ is trivialized by $f$, then it follows that $V$ is essentially finite with étale monodromy gerbe in EFin $(\operatorname{Vect}(\mathcal{X}))$. Indeed, replacing $f$ by a Galois closure one can assume that $f$ is an étale Galois cover. This case is exactly [TZ3, Lemma 1.4].

Lemma 1.17. Let $T^{\prime \prime} \stackrel{a}{\longrightarrow} T$ and $T^{\prime} \stackrel{b}{\longrightarrow} T$ be two maps of affine group schemes over $k$, and let

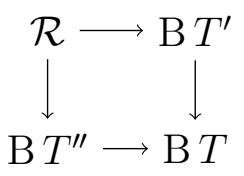


be the corresponding 2-Cartesian diagram. Then the following two hold.

(1) The functor $\Psi: \mathrm{B}\left(T^{\prime \prime} \times_{T} T^{\prime}\right) \longrightarrow \mathcal{R}$ mapping a $T^{\prime \prime} \times_{T} T^{\prime}$-torsor to the associated $T^{\prime \prime}$ - and $T^{\prime}$ - torsors is fully faithful and it is an equivalence if and only if the map $T^{\prime} \times T^{\prime \prime} \longrightarrow T,\left(t^{\prime}, t^{\prime \prime}\right) \mapsto b\left(t^{\prime}\right) a\left(t^{\prime \prime}\right)$ is an fpqc epimorphism (e.g. if a or $b$ is surjective). In this case a quasi-inverse is obtained by mapping an object of $\mathcal{R}$ given by torsors $P^{\prime \prime}, P^{\prime}, P$ under $T^{\prime \prime}, T^{\prime}, T$ respectively and equivariant maps $P^{\prime} \longrightarrow P$ and $P^{\prime \prime} \longrightarrow P$ to the fiber product $P^{\prime \prime} \times_{P} P^{\prime}$.

(2) If $T^{\prime \prime}=$ Spec $k$, so that $\mathrm{B} T^{\prime \prime}=$ Spec $k$, and $T^{\prime} \longrightarrow T$ is injective, then $\mathcal{R}=T / T^{\prime}$, where $T / T^{\prime} \longrightarrow \mathrm{B} T^{\prime}$ is induced by the $T^{\prime}$-torsor $T \longrightarrow T / T^{\prime}$. In particular, if $T^{\prime}$ is a finite subgroup of $T$, then $\mathrm{B} T^{\prime} \longrightarrow \mathrm{B} T$ is an affine map.

Proof. The functor $\Psi$ maps the trivial torsor to $\left(T^{\prime \prime}, T^{\prime}, \mathrm{id}\right) \in \mathcal{R}(k)$. A direct computation shows that the sheaf of automorphisms of this object is exactly $T^{\prime \prime} \times_{T} T^{\prime}$ (via $\Psi$ ). This means that $\Psi$ is an equivalence onto the full-substack $\mathcal{R}^{\prime}$ of $\mathcal{R}$ of objects locally isomorphic to $\left(T^{\prime \prime}, T^{\prime}\right.$, id). Thus we have to understand when $\mathcal{R}^{\prime}=\mathcal{R}$. All objects of $\mathcal{R}$ are locally isomorphic to an object of the form $\left(T^{\prime \prime}, T^{\prime}, c\right) \in \mathcal{R}(U)$ where $U$ is an affine scheme and $c \in$ $T(U)$ is thought of as multiplication on the left $T \longrightarrow T$. An isomorphism $\left(T^{\prime \prime}, T^{\prime}, 1\right) \longrightarrow$ $\left(T^{\prime \prime}, T^{\prime}, c\right)$ is given by $t^{\prime \prime} \in T^{\prime \prime}(U)$ and $t^{\prime} \in T^{\prime}(U)$ such that $c a\left(t^{\prime \prime}\right)=b\left(t^{\prime}\right)$. Thus $\left(T^{\prime \prime}, T^{\prime}, c\right)$ is locally isomorphic to $\left(T^{\prime \prime}, T^{\prime}, 1\right)$ if and only if $c$ is in the (fpqc) image of $T^{\prime} \times T^{\prime \prime} \longrightarrow T$. The last claim of (1) follows because if $\bar{P}$ is a $T^{\prime \prime} \times_{T} T^{\prime}$-torsor inducing torsors $P^{\prime \prime}, P^{\prime}, P$ under $T^{\prime \prime}, T^{\prime}, T$ respectively then the commutative diagram

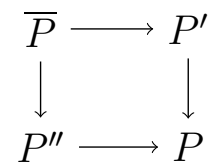

is automatically Cartesian: locally, after choosing a section of $\bar{P}$, the above diagram is the one yielding $T^{\prime \prime} \times_{T} T^{\prime}$. Notice that the $T^{\prime \prime} \times_{T} T^{\prime}$-space given in the last part of (1) is not a torsor in general because it may fail to have sections locally.

For $(2), \mathcal{R}$ is the sheaf of $T^{\prime}$-torsors $P$ together with an equivariant map $P \longrightarrow T$, which is represented by $T / T^{\prime}$.

Remark 1.18. If $f: \mathcal{Y} \longrightarrow \mathcal{X}$ is a cover of algebraic stacks then $f_{*}$ preserves vector bundles. Moreover since $f_{*}$ is exact we have

$$
\mathrm{H}^{i}(\mathcal{Y}, E)=\mathrm{H}^{i}\left(\mathcal{X}, f_{*} E\right) \text { for all } i \geqslant 0, E \in \mathrm{Q} \operatorname{Coh}(\mathcal{Y}) .
$$

In particular, if $\mathcal{X}$ is pseudo-proper over $k$, then $\mathcal{Y}$ is pseudo-proper over $k$. Moreover we will often use also the following property: if for all vector bundles $E$ on $\mathcal{X}$ one has $\operatorname{dim}_{k} \mathrm{H}^{1}(E)<\infty$ then the same holds for vector bundles on $\mathcal{Y}$.

Lemma 1.19 ([TZ2, Lemma 2.5]). Let $\mathcal{X}$ be an algebraic stack over a field $k$, of positive characteristic, such that $\operatorname{dim}_{k} \mathrm{H}^{1}(X, E)<\infty$ for all vector bundles $E$ on $\mathcal{X}$. Let

$$
\mathcal{G}_{0} \longrightarrow \mathcal{G}_{1} \longrightarrow \cdots \longrightarrow \mathcal{G}_{N-1} \longrightarrow \mathcal{G}_{N}=0
$$


be a sequence of surjective maps of quasi-coherent sheaves on $\mathcal{X}$ such that $\operatorname{Ker}\left(\mathcal{G}_{l-1} \longrightarrow \mathcal{G}_{l}\right)$ is free of finite rank for all $1 \leqslant l \leqslant N$. Then there exists a surjective cover $f: \mathcal{X}^{\prime} \longrightarrow \mathcal{X}$ such that $f^{*} \mathcal{G}_{l}$ is free of finite rank for all $l$.

Remark 1.20 ([TZ1, Example 1.5, Corollary 1.7]). Let $\Gamma$ be a finite stack or an affine gerbe over $k$. For all fibered categories $\mathcal{Z}$ over $k$, the pullback of vector bundles establishes an equivalence of categories between $\operatorname{Hom}_{k}(\mathcal{Z}, \Gamma)$ and the groupoid of functors $\vee$ ect $(\Gamma) \longrightarrow$ $\operatorname{Vect}(\mathcal{Z})$ which are $k$-linear, monoidal and preserves short exact sequences in the category of quasi-coherent sheaves.

Remark 1.21. Let us comment on the relationship between the essentially finite vector bundles on a fibered category $\mathcal{X}$ over $k$ and the vector bundles which are pullbacks from a finite stack. When $\mathcal{X}$ is inflexible and pseudo-proper, these two notions agree as a consequence of [BV]. One of the key observations in [BV] is that if $\Gamma$ is a finite stack over $k$ and $V \in \operatorname{Vect}(\Gamma)$, then $V$ is essentially finite. More precisely, there is a finite vector bundle $E$ on $\Gamma$ and an exact sequence in $\operatorname{Coh}(\Gamma)$

$$
0 \longrightarrow V \longrightarrow E^{\oplus a} \longrightarrow E^{\oplus b} \longrightarrow E^{\prime} \longrightarrow 0
$$

for some $a, b \in \mathbb{N}$ and $E^{\prime} \in \operatorname{Vect}(\Gamma)$. In particular, if $\phi: \mathcal{X} \longrightarrow \Gamma$ is any map from a fibered category, then $\phi^{*} V$ is an essentially finite vector bundle on $\mathcal{X}$. The proof of this fact is the same as that of [BV, Lemma 7.15] together with the following clarification. First we can assume that $\Gamma$ is connected. Let $\rho: T \longrightarrow \Gamma$ be a surjective cover from a finite connected $k$-scheme $T$. The direct image $E=\rho_{*} \mathcal{O}_{T}$ is finite by [BV, Lemma 7.15]. Since the cokernel of $V \longrightarrow \rho_{*} \rho^{*} V \simeq E^{\oplus \operatorname{rk} V}$ is a vector bundle one can easily construct the above sequence.

Now let $\mathcal{X}$ be a fibered category and $V \in \operatorname{Vect}(\mathcal{X})$. If $V$ is essentially finite, one might argue that there is a homomorphism between two finite vector bundles $q: E_{1} \longrightarrow E_{2}$ whose kernel is $V$. This is actually misleading. Since the definition of essentially finite in the category $\operatorname{Vect}(\mathcal{X})$ is intrinsic to this category, $V$ has to be a kernel of $q$ inside the category $\operatorname{Vect}(\mathcal{X})$. This does not imply that $V$ coincides with the kernel $\mathcal{K}$ of $q$ in $\mathrm{QCoh}(\mathcal{X})$. This equality holds if $\mathcal{X}$ is pseudo-proper and inflexible. If $\mathcal{X}$ has the resolution property, that is all quasi-coherent sheaves are quotients of sums of vector bundles (e.g. when $\mathcal{X}$ is a quasi-projective scheme or a smooth separated scheme), and if the kernel $V$ exists in the category $\operatorname{Vect}(\mathcal{X})$, then $V=\mathcal{K}$.

In order to avoid the above-mentioned issue, if $\mathcal{X}$ is pseudo-proper but not inflexible it seems to us that the "correct" essentially finite vector bundles to use are vector bundles coming from a finite stack or at least that are kernel in $\mathrm{QCoh}(\mathcal{X})$ of a map of finite vector bundles. Although this is not an intrinsic notion it would be a good working definition. This should also explain why Lemma 2.13 and Lemma 2.11 should be understood as results assuring that pushforward preserves essentially finite vector bundles. In any case in the present paper we consider essentially finite vector bundles only on pseudo-proper and inflexible fibered categories, so we maintain the notion of essentially finite in Definition 1.5 .

There is a partial converse to the fact that vector bundles coming from finite stacks are essentially finite. If $\mathcal{X}$ is a fibered category over $k$ with $\operatorname{dim}_{k} \mathrm{H}^{0}\left(\mathcal{O}_{\mathcal{X}}\right)<\infty$ and $V \in \operatorname{Vect}(\mathcal{X})$ 
is a finite vector bundle, then there exist a map $\phi: \mathcal{X} \longrightarrow \Phi$ to a finite stack and $W \in$ $\operatorname{Vect}(\Phi)$ such that $V \simeq \phi^{*} W$. This is essentially proved in [BV, p. 23] (just after the proof of Lemma 7.11). We recall here the construction for the convenience of the reader. We can assume that $V$ has rank $r$ and take $f \neq g \in \mathbb{N}[x]$ such that $f(V) \simeq g(V)$. The group $\mathrm{GL}_{r}$ acts on the scheme $I=\underline{\mathrm{IsO}}\left(f\left(k^{r}\right), g\left(k^{r}\right)\right)=\mathrm{GL}_{N}$ with $N=f(r)=g(r)$. The isomorphism $f(V) \simeq g(V)$ gives a factorization of the vector bundle $V: \mathcal{X} \longrightarrow \mathrm{B} \mathrm{GL}_{r}$ through $\left[I / \mathrm{GL}_{r}\right]$ and we have Cartesian diagrams

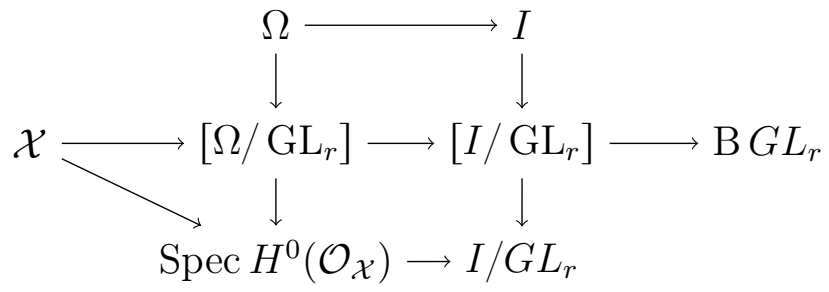

Here we are using that $I \longrightarrow I / \mathrm{GL}_{r}$ is a geometric quotient and $I / \mathrm{GL}_{r}$ is affine because $\mathrm{GL}_{r}$ is geometrically reductive. Thus we must show that $\Phi=\left[\Omega / \mathrm{GL}_{r}\right]$ is a finite stack. As the geometric fibers of $I \longrightarrow I / \mathrm{GL}_{r}$ consist (topologically) of one orbit and $\mathrm{H}^{0}\left(\mathcal{O}_{\mathcal{X}}\right)$ is a finite $k$-algebra one sees that $\Phi(\bar{k})$ has finitely many isomorphism classes. The action of $\mathrm{GL}_{r}$ on $I$ has finite stabilizers by [BV, Lemma 7.12] and hence it follows that the diagonal of $\Phi$ is quasi-finite. By [BV, Proposition 4.2] it follows that $\Phi$ is a finite stack.

Lemma 1.22. Let $\mathcal{X}$ be a fibered category which admits an fpqc-atlas from a scheme, and let $u: \mathcal{X} \longrightarrow \Gamma$ be a quasi-compact and quasi-separated map, where $\Gamma$ is an affine gerbe. Then $u^{*}: \operatorname{Vect}(\Gamma) \longrightarrow \operatorname{Vect}(\mathcal{X})$ is fully faithful if and only if $u^{\#}: \mathcal{O}_{\Gamma} \longrightarrow u_{*} \mathcal{O}_{\mathcal{X}}$ is an isomorphism.

Proof. For $V, V^{\prime} \in \operatorname{Vect}(\Gamma)$ the composition

$$
\operatorname{Hom}_{\Gamma}\left(V, V^{\prime}\right) \stackrel{u^{*}}{\longrightarrow} \operatorname{Hom}_{\mathcal{X}}\left(u^{*} V, u^{*} V^{\prime}\right) \simeq \operatorname{Hom}_{\Gamma}\left(V, u_{*} u^{*} V^{\prime}\right) \simeq \operatorname{Hom}_{\Gamma}\left(V, V^{\prime} \otimes u_{*} \mathcal{O}_{\mathcal{X}}\right)
$$

is the map induced by $u^{\#}: \mathcal{O}_{\Gamma} \longrightarrow u_{*} \mathcal{O}_{\mathcal{X}}$. In particular, if this map is an isomorphism then $u^{*}: \operatorname{Vect}(\Gamma) \longrightarrow \operatorname{Vect}(\mathcal{X})$ is fully faithful.

Conversely, setting $V^{\prime}=\mathcal{O}_{\Gamma}$ above, we have that the homomorphism

$$
\operatorname{Hom}_{\Gamma}\left(V, \mathcal{O}_{\Gamma}\right) \longrightarrow \operatorname{Hom}_{\Gamma}\left(V, u_{*} \mathcal{O}_{\mathcal{X}}\right)
$$

induced by $u^{\#}$ is an isomorphism for all vector bundles $V$ over $\Gamma$. Since $u_{*} \mathcal{O}_{\mathcal{X}}$ is a quasicoherent sheaf, there is a surjective map $a: \oplus V_{i} \rightarrow u_{*} \mathcal{O}_{\mathcal{X}}$, where $V_{i}$ are vector bundles on $\Gamma$ [De, p. 132, Corollary 3.9]. The above isomorphism produces a map $\oplus V_{i} \longrightarrow \mathcal{O}_{\Gamma}$ whose composition with $u^{\#}$ is $a$, so we get that $u^{\#}$ is surjective. Since $u: \mathcal{X} \longrightarrow \Gamma$ is faithfully flat we also have that $u^{\#}$ injective.

Remark 1.23. If $\mathcal{X}$ is a pseudo-proper and inflexible algebraic stack over $k$ and if $\alpha: \mathcal{X} \longrightarrow$ $\Pi_{\mathcal{X}}^{\mathrm{N}}$ is the structure map of the Nori fundamental gerbe, then using Theorem 1.10 and Lemma 1.22 we conclude that $\alpha_{*} \mathcal{O}_{\mathcal{X}} \simeq \mathcal{O}_{\Pi_{\mathcal{X}}^{\mathrm{N}}}$. The same holds for the Nori étale fundamental gerbe. 


\section{Essentially finite covers AND their Nori Gerbes}

Let $k$ be a base field. In this section we study the notion of an essentially finite cover, which generalizes the notion of torsor under a finite group scheme. Moreover we are going to prove Theorem I and Corollary I.

Recall that an essentially finite cover $f: \mathcal{Y} \longrightarrow \mathcal{X}$ of fibered categories is a cover such that $f_{*} \mathcal{O}_{\mathcal{Y}}$ is essentially finite as an object of $\operatorname{Vect}(\mathcal{X})$ (see Definition 1.11).

First observe that a torsor under a finite group scheme is an essentially finite cover. Indeed, let $f: \mathcal{Y} \longrightarrow \mathcal{X}$ be a torsor under a finite group scheme $G$ over $k$ corresponding to $u: \mathcal{X} \longrightarrow \mathrm{B} G$. Then $u^{*}(k[G]) \simeq f_{*} \mathcal{O}_{\mathcal{Y}}$, where $k[G]$ is the regular representation. Applying $[\mathrm{BV}$, Lemma 7.15] to Spec $k \longrightarrow \mathrm{B} G$ we see that $k[G]$ is finite in $\operatorname{Vect}(\mathrm{B} G)=\operatorname{Rep} G$ and thus $f_{*} \mathcal{O}_{\mathcal{Y}}$ is a finite vector bundle.

Proposition 2.1. Let $\mathcal{X}$ be a pseudo-proper and inflexible fibered category over $k$. Then there is an equivalence of categories

$$
\left\{\begin{array}{c}
\text { Stacks } \text { finite } \\
\text { over } \Pi_{\mathcal{X}}^{\mathrm{N}}
\end{array}\right\} \stackrel{\Phi}{\longrightarrow}\left\{\begin{array}{c}
\text { Essentially finite } \\
\text { covers of } \mathcal{X}
\end{array}\right\}
$$

where $\Phi$ is the pullback along $\mathcal{X} \longrightarrow \Pi_{\mathcal{X}}^{\mathrm{N}}$. If moreover $x \in \mathcal{X}(k)$ is a rational point, then there is an equivalence

$$
\left\{\begin{array}{c}
\text { Essentially finite } \\
\text { covers of } \mathcal{X}
\end{array}\right\} \stackrel{\Psi}{\longrightarrow}\left\{\begin{array}{l}
\text { Finite } k \text {-schemes with } \\
\text { an action of } \pi^{\mathrm{N}}(\mathcal{X}, x)
\end{array}\right\}
$$

where $\Psi$ is the pullback along Spec $k \stackrel{x}{\longrightarrow} \mathcal{X}$. Furthermore, $\Psi$ extends the correspondence between pointed Nori-reduced torsors of $\mathcal{X}$ and quotient group schemes of $\pi^{\mathrm{N}}(\mathcal{X}, x)$.

Proof. The functor $\Phi$ is the equivalence mapping ring objects of $\operatorname{Vect}\left(\Pi_{\mathcal{X}}^{\mathrm{N}}\right)$ to ring objects of $\operatorname{EFin}(\operatorname{Vect}(X))$. If $x \in \mathcal{X}(k)$, then $\Pi_{\mathcal{X}}^{\mathrm{N}}=\mathrm{B} \pi^{\mathrm{N}}(\mathcal{X}, x)$, and the ring objects of $\operatorname{Vect}\left(\Pi_{\mathcal{X}}^{\mathrm{N}}\right)=$ $\operatorname{Rep} \pi^{\mathrm{N}}(\mathcal{X}, x)$ are precisely the finite $k$-algebras with an action of $\pi^{\mathrm{N}}(\mathcal{X}, x)$. This easily implies that $\Psi \circ \Phi$ is an equivalence. The last claim follows by construction.

Lemma 2.2. Let $\mathcal{X}$ be a pseudo-proper and inflexible fibered category over $k$ and let $f: \mathcal{Y} \longrightarrow \mathcal{X}$ an essentially finite cover; let $u: \mathcal{X} \longrightarrow \Gamma$ be the monodromy gerbe of $f_{*} \mathcal{O}_{\mathcal{Y}} \in \operatorname{EFin}(\operatorname{Vect}(\mathcal{X}))$. Then there exist unique covers $\Delta \longrightarrow \Gamma$ and $\Pi \longrightarrow \Pi_{\mathcal{X} / k}^{\mathrm{N}}$ which make the following diagrams

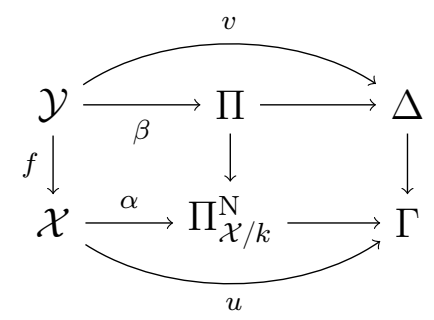


Cartesian, and we also have $\beta_{*} \mathcal{O}_{\mathcal{Y}} \simeq \mathcal{O}_{\Pi}, v_{*} \mathcal{O}_{\mathcal{Y}} \simeq \mathcal{O}_{\Delta}$. If $\mathcal{Y}$ is inflexible then $\Pi, \Delta$ are affine gerbes.

Proof. The multiplication map of $f_{*} \mathcal{O}_{\mathcal{Y}}$ and its unit map lie in $\operatorname{Vect}(\Gamma) \subseteq \operatorname{Vect}(\mathcal{X})$ and therefore determine a cover

$$
\Delta \longrightarrow \Gamma
$$

extending $f$ as claimed in the statement. Uniqueness of the extension follows from the fact that $\operatorname{Vect}(\Gamma) \longrightarrow \operatorname{Vect}(\mathcal{X})$ is fully faithful. As $u$ is Nori reduced we have $u_{*} \mathcal{O}_{\mathcal{X}} \simeq \mathcal{O}_{\Gamma}$ (see Lemma 1.22 or [BV, Lemma 7.11, pp. 22]). Since $\Delta \longrightarrow \Gamma$ is flat we also conclude that $v_{*} \mathcal{O}_{\mathcal{Y}} \simeq \mathcal{O}_{\Delta}$. The cover $\Pi \longrightarrow \Pi_{\mathcal{X} / k}^{\mathrm{N}}$ and its uniqueness plus the fact $\beta_{*} \mathcal{O}_{\mathcal{Y}} \simeq \mathcal{O}_{\Pi}$ are obtained in exactly the same way.

Assume now that $\mathcal{Y}$ is inflexible. By definition, $\mathcal{Y} \longrightarrow \Delta$ factors through a finite gerbe $\Delta^{\prime}$, which can be chosen as closed substack $\Delta^{\prime} \subseteq \Delta$. But $v_{*} \mathcal{O}_{\mathcal{Y}} \simeq \mathcal{O}_{\Delta}$ which implies that $\Delta=\Delta^{\prime}$ as required. Notice that, since $\Pi_{\mathcal{X} / k}^{\mathrm{N}} \longrightarrow \Gamma$ is a quotient, the stack $\Delta$ is a gerbe if and only if $\Pi$ is a gerbe. This completes the proof.

Proof of Theorem II. The existence of the diagram is clear: firstly $\Delta, \Gamma, u$ are by Lemma 2.2 , then $\operatorname{Spec}(k) \longrightarrow \Delta$ is the image of $y \in \mathcal{Y}(k)$, and finally $\mathcal{P}_{f}$ is defined as a product. Since $u: \mathcal{X} \longrightarrow \Gamma=\mathrm{B} G_{f}$ is Nori-reduced so is the $G_{f}$-torsor $\mathcal{P}_{f} \longrightarrow \mathcal{X}$. The claim about the monodromy gerbe of $\pi_{*} \mathcal{O}_{\mathcal{P}_{f}}$ follows from the following fact: if $G$ is a finite group scheme over $k$, the regular representation $k[G]$ generates $\operatorname{Rep} G$ because every finite $G$-representation is a subobject of some $k[G]^{n}$.

The morphism $\lambda$ is faithfully flat if and only if Spec $k \longrightarrow \Delta$ is faithfully flat. This is the case if and only if $\Delta$ is a gerbe. In such a situation we have $\Delta=\mathrm{B} H$, where $H$ is a subgroup of $G_{f}$ and $\mathcal{P}_{f} \stackrel{\lambda}{\longrightarrow} \mathcal{Y}$ is an $H$-torsor. The map $u^{\prime}: \mathcal{Y} \longrightarrow \Delta=\mathrm{B} H$ is Nori-reduced because $u_{*}^{\prime} \mathcal{O}_{\mathcal{Y}} \simeq \mathcal{O}_{\Delta}$.

The factorization $\lambda=\eta \circ \lambda^{\prime}$ arises from the factorization Spec $k \longrightarrow \Delta^{\prime} \longrightarrow \Delta$, where $\Delta^{\prime}$ is a subgerbe of the finite stack $\Delta$.

For the last statement, $h$ induces an inclusion $\mathcal{O}_{\mathcal{Y}} \subset h_{*} \mathcal{O}_{\mathcal{T}}$, and as $f$ is affine, an inclusion $f_{*} \mathcal{O}_{\mathcal{Y}} \subset g_{*} \mathcal{O}_{\mathcal{T}}$. If we denote by $\left\langle g_{*} \mathcal{O}_{\mathcal{T}}\right\rangle$ (respectively, $\left\langle\pi_{*} \mathcal{O}_{\mathcal{P}_{f}}\right\rangle$ ) the full Tannakian subcategory of $\operatorname{EFin}(\operatorname{Vect}(\mathcal{X}))$ generated by the object $g_{*} \mathcal{O}_{\mathcal{T}}$ (respectively, $\pi_{*} \mathcal{O}_{\mathcal{P}_{f}}$ ), one gets the following diagram with 2-Cartesian square:

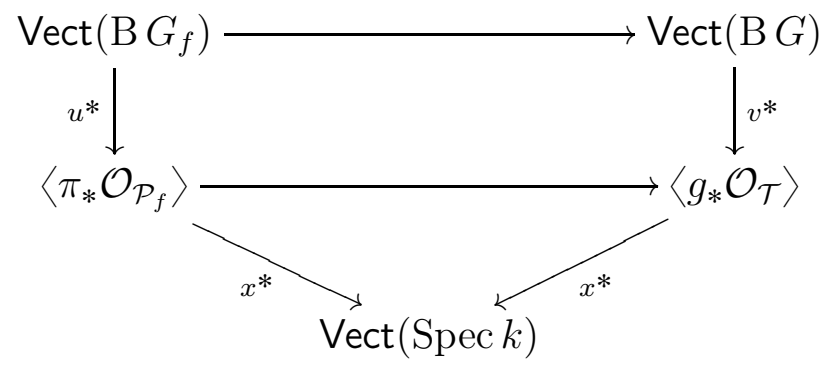

where $v: \mathcal{X} \longrightarrow \mathrm{B} G$ corresponds to the torsor $g: \mathcal{T} \longrightarrow \mathcal{X}$, the horizontal arrows are inclusions and the vertical arrows are equivalences. As the torsors are pointed above $x$, 
the functors $x^{*} \circ u^{*}$ and $x^{*} \circ v^{*}$ are equivalent to the forgetful functors. Therefore, the commutativity of this last diagram proves the existence of a surjective morphism $\varphi: G \longrightarrow$ $G_{f}$ such that $u^{*} \simeq v^{*} \circ \varphi^{*}: \operatorname{Vect}\left(\mathrm{B} G_{f}\right) \longrightarrow \operatorname{Vect}(\mathcal{X})$. This implies that $u=\varphi^{\prime} \circ v$, where $\varphi^{\prime}: \mathrm{B} G \longrightarrow \mathrm{B} G_{f}$ is the morphism of gerbes induced by $\varphi$.

Definition 2.3. Let $\mathcal{X}$ be a pseudo-proper, inflexible fibered category over $k$ with a base point $x \in \mathcal{X}(k)$, and let $f:(\mathcal{Y}, y) \longrightarrow(\mathcal{X}, x)$ be an essentially finite cover. The Galois closure of $f$ is the pointed torsor $\mathcal{P}_{f} \longrightarrow \mathcal{X}$ with the pointed map $\mathcal{P}_{f} \longrightarrow \mathcal{Y}$ constructed in Theorem II.

Definition 2.4. Let $\mathcal{X}$ be a pseudo-proper, inflexible fibered category over $k$. We call an essentially finite cover $f: \mathcal{Y} \longrightarrow \mathcal{X}$ inflexible if $\mathcal{Y}$ is inflexible.

Remark 2.5. Let $\mathcal{X}$ be a pseudo-proper and inflexible algebraic stack of finite type over a field $k$ of positive characteristic, such that $\operatorname{dim}_{k} \mathrm{H}^{1}(\mathcal{X}, E)<\infty$ for all vector bundles $E$ on $\mathcal{X}$. Let $f: \mathcal{Y} \longrightarrow \mathcal{X}$ be a Nori-reduced torsor. Then by Theorem I (which will be proved later) $f$ is inflexible.

Lemma 2.6. Let $\mathcal{X}$ be a pseudo-proper, inflexible fibered category with a rational point $x \in \mathcal{X}(k)$. Let $f: \mathcal{Y} \longrightarrow \mathcal{X}$ be an inflexible essentially finite cover equipped with a rational point $y \in \mathcal{Y}(k)$ mapping to $x$. Then the Galois closure $\mathcal{P}_{f}$ of $f$ satisfies:

- The $G_{f}$-torsor $\mathcal{P}_{f}$ is Nori-reduced.

- For any pointed Nori-reduced torsor $g:(\mathcal{T}, t) \longrightarrow(\mathcal{X}, x)$ under a finite group scheme $G$, and any pointed $\mathcal{X}$-morphism $h:(\mathcal{T}, t) \rightarrow(\mathcal{Y}, y)$, there is a unique factorization $h=\lambda \circ j$, where $j:(\mathcal{T}, t) \longrightarrow\left(\mathcal{P}_{f}, p\right)$ is equivariant with respect to a surjective homomorphism $G \longrightarrow G_{f}$.

Proof. Using Lemma 2.2 one can easily show that when $\mathcal{Y}$ is inflexible any map from an essentially finite cover $\mathcal{T}$ to $\mathcal{Y}$ is faithfully flat. In view of Theorem II (4) we can conclude the proof.

Here are some technical lemmas which will be used in proving Theorem I.

Lemma 2.7. Consider a 2-Cartesian diagram

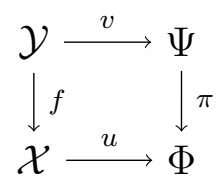

where $\mathcal{X}, \mathcal{Y}, \Phi, \Psi$ are fibered categories over $k$ which admit fpqc-atlases from schemes, and $u, \pi$ are faithfully flat where $\pi$ is affine and $u$ is quasi-compact and quasi-separated such that $u_{*} \mathcal{O}_{\mathcal{X}} \simeq \mathcal{O}_{\Phi}$. Then $v_{*} \mathcal{O}_{\mathcal{Y}} \simeq \mathcal{O}_{\Psi}$, and the two functors $u^{*}: \operatorname{Vect}(\Phi) \longrightarrow \operatorname{Vect}(\mathcal{X})$ and $v^{*}: \operatorname{Vect}(\Psi) \longrightarrow \operatorname{Vect}(\mathcal{Y})$ are fully faithful.

$A$ vector bundle $V \in \operatorname{Vect}(\mathcal{X})$ lies in the essential image of $u^{*}: \operatorname{Vect}(\Phi) \longrightarrow \operatorname{Vect}(\mathcal{X})$ if and only if $f^{*} V$ comes from a vector bundle on $\Psi$.

If $\pi$ is a surjective cover, a vector bundle $V \in \operatorname{Vect}(\mathcal{Y})$ lies in the essential image of $v^{*}: \operatorname{Vect}(\Psi) \longrightarrow \operatorname{Vect}(\mathcal{Y})$ if and only if $f_{*} V$ comes from a vector bundle on $\Phi$. 
Proof. By [BV, Lemma 7.17] and flat base change it follows that

- $v_{*} \mathcal{O}_{\mathcal{Y}} \simeq \mathcal{O}_{\Psi}$,

- $u^{*}: \operatorname{Vect}(\Phi) \longrightarrow \operatorname{Vect}(\mathcal{X})$ and $v^{*}: \operatorname{Vect}(\Psi) \longrightarrow \operatorname{Vect}(\mathcal{Y})$ are fully faithful.

Denote by $\mathcal{D}$ and $\mathcal{C}$ the essential images of these $u^{*}$ and $v^{*}$ respectively.

Let $V \in \operatorname{Vect}(\mathcal{X})$. We must show that $V \in \mathcal{D}$ if and only if $f^{*} V \in \mathcal{C}$. The "only if" part is clear. Conversely, suppose that $f^{*} V=v^{*} W$ with $W \in \operatorname{Vect}(\Psi)$, and consider the canonical homomorphism $u^{*} u_{*} V \longrightarrow V$; pulling back by $f$ one gets

$$
v^{*} v_{*}\left(f^{*} V\right)=v^{*} v_{*}\left(v^{*} W\right) \longrightarrow v^{*} W=f^{*} V .
$$

This homomorphism is an isomorphism because $v_{*} \mathcal{O}_{\mathcal{Y}} \simeq \mathcal{O}_{\Psi}$. As $f$ is faithfully flat, one concludes that $V \simeq u^{*} u_{*} V$, and as $u$ is faithfully flat, it follows that $u_{*} V$ is a vector bundle. Thus we have $V \in \mathcal{D}$.

Assume now that $\pi$ is a surjective cover; consequently $f$ is also a surjective cover. In particular, $\pi_{*}$ and $f_{*}$ send vector bundles to vector bundles. Given $V \in \operatorname{Vect}(\mathcal{Y})$ we must show that $V \in \mathcal{C}$ if and only if $f_{*} V \in \mathcal{D}$. The "only if" part is easy: if $W \in \operatorname{Vect}(\Psi)$ then $f_{*}\left(v^{*} W\right) \simeq u^{*} \pi_{*} W$ because $\pi$ is affine.

For the converse, assume that $f_{*} V \in \mathcal{D}$, meaning $f_{*} V$ comes from a vector bundle on $\Phi$. Since $u_{*} \mathcal{O}_{\mathcal{X}} \simeq \mathcal{O}_{\Phi}$ it follows that $u_{*}\left(f_{*} V\right)$ is a vector bundle and the canonical homomorphism $u^{*} u_{*}\left(f_{*} V\right) \longrightarrow\left(f_{*} V\right)$ is an isomorphism. This homomorphism can also be obtained by applying $f_{*}$ to the canonical homomorphism $v^{*} v_{*} V \longrightarrow V$. Since $f$ is affine this means that the previous homomorphism is an isomorphism. To conclude that $V \in \mathcal{C}$ it suffices to show that $v_{*} V$ is a vector bundle. But $v$ is faithfully flat and $v^{*}\left(v_{*} V\right)$ is a vector bundle. Now by descent it follows that $v_{*} V$ is also a vector bundle.

Remark 2.8. Consider a $G$-torsor $f: \mathcal{Y} \rightarrow \mathcal{X}$ for an affine group scheme $G$, where $\mathcal{X}$ is a quasi-compact and quasi-separated algebraic stack, and the corresponding 2-Cartesian diagram

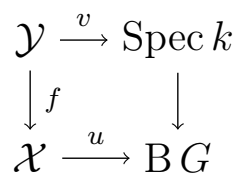

We see that $\mathrm{H}^{0}\left(\mathcal{O}_{\mathcal{Y}}\right)=k$ if and only if $u_{*} \mathcal{O}_{\mathcal{X}} \simeq \mathcal{O}_{\mathrm{B} G}$. In this case, applying Lemma 2.7, we conclude that $u^{*}: \operatorname{Vect}(\mathrm{B} G) \longrightarrow \operatorname{Vect}(\mathcal{X})$ is fully faithful with essential image the category of vector bundles $V$ such that $f^{*} V$ is trivial.

Lemma 2.9 ([No2, p. 264, Lemma 1]). Let $\mathcal{X}$ be a quasi-compact and quasi-separated algebraic algebraic stack and

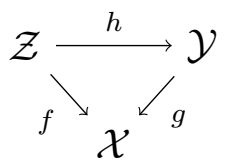

a 2-commutative diagram, where $f$ and $g$ are torsors for affine group schemes $G$ and $H$ respectively. Suppose that $\mathrm{H}^{0}\left(\mathcal{O}_{\mathcal{Z}}\right)=k$. Then there exists a homomorphism $\varphi: G \longrightarrow H$ inducing $h$. 
Moreover, $h$ is faithfully flat if and only if $\varphi: G \longrightarrow H$ is faithfully flat, in which case $h: \mathcal{Z} \longrightarrow \mathcal{Y}$ is a torsor for the kernel of $\varphi$. If $H / \operatorname{Im}(\varphi)$ is affine (e.g. if $H$ or $G$ are finite) then this is also equivalent to the statement that $\mathrm{H}^{0}\left(\mathcal{O}_{\mathcal{Y}}\right)=k$.

Proof. Consider the morphisms $u: \mathcal{X} \longrightarrow \mathrm{B} G$ and $v: \mathcal{X} \longrightarrow \mathrm{B} H$ corresponding to the torsors $f$ and $g$ respectively. Since $\mathrm{H}^{0}\left(\mathcal{O}_{\mathcal{Z}}\right)=k$ we have $u_{*} \mathcal{O}_{\mathcal{X}} \simeq \mathcal{O}_{\mathrm{B} G}$, and hence by 1.22 the pullback functor $u^{*}: \operatorname{Vect}(\mathrm{B} G) \longrightarrow \operatorname{Vect}(\mathcal{X})$ is fully faithful. The objects of the essential image of $v^{*}: \operatorname{Vect}(\mathrm{B} H) \longrightarrow \operatorname{Vect}(\mathcal{X})$ are trivialized by $g$ and thus by $f$. From Remark 2.8 we obtain a factorization

$$
v^{*}: \operatorname{Vect}(\mathrm{B} H) \longrightarrow \operatorname{Vect}(\mathrm{B} G) \subseteq \operatorname{Vect}(\mathcal{X})
$$

which, by Tannakian duality, is induced by a factorization $v: \mathcal{X} \stackrel{u}{\longrightarrow} \mathrm{B} G \stackrel{\gamma}{\longrightarrow} \mathrm{B} H$. Consider the 2-Cartesian diagrams

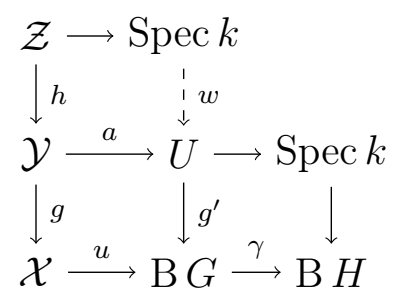

We claim that there exists a dashed arrow $w$ as above making the upper diagram 2Cartesian. This would imply that the functor $\gamma: \mathrm{B} G \longrightarrow \mathrm{B} H$ is induced by a group homomorphism $\varphi: G \longrightarrow H$.

Set $f^{\prime}:$ Spec $k \longrightarrow \mathrm{B} G$. Consider the map of $\mathcal{O}_{\mathcal{X}}$-algebras $\lambda: g_{*} \mathcal{O}_{\mathcal{Y}} \longrightarrow f_{*} \mathcal{O}_{\mathcal{Z}}$. Applying $u_{*}$ to $\lambda$ and using $a_{*} \mathcal{O}_{\mathcal{Y}} \simeq \mathcal{O}_{U}, \mathrm{H}^{0}\left(\mathcal{O}_{\mathcal{Z}}\right)=k$, we get a map

$$
g^{\prime}{ }_{*} \mathcal{O}_{U} \cong g^{\prime}{ }_{*} a_{*} \mathcal{O}_{\mathcal{Y}} \cong u_{*} g_{*} \mathcal{O}_{\mathcal{Y}} \longrightarrow u_{*} f_{*} \mathcal{O}_{\mathcal{Z}} \cong f^{\prime}{ }_{*} \mathcal{O}_{\text {Spec }(k)} \text {. }
$$

Applying $\operatorname{Spec}_{\mathrm{B} G}(-)$ on both sides we get the arrow $w$.

To prove that $h$ is the pullback of $w$ we just have to show that the adjunction maps

$$
u^{*} u_{*} g_{*} \mathcal{O}_{\mathcal{Y}} \longrightarrow g_{*} \mathcal{O}_{\mathcal{Y}} \quad \text { and } \quad u^{*} u_{*} f_{*} \mathcal{O}_{\mathcal{Z}} \longrightarrow f_{*} \mathcal{O}_{\mathcal{Z}}
$$

are isomorphisms. But the adjunction map for $g_{*} \mathcal{O}_{\mathcal{Y}}$ coincides with the following composition:

$$
u^{*} u_{*} g_{*} \mathcal{O}_{\mathcal{Y}} \cong u^{*} g^{\prime}{ }_{*} a_{*} \mathcal{O}_{\mathcal{Y}} \cong u^{*} g^{\prime}{ }_{*} \mathcal{O}_{U} \cong g_{*} \mathcal{O}_{\mathcal{Y}}
$$

which is an isomorphism, and the same is true for $f_{*} \mathcal{O}_{\mathcal{Z}}$.

The map $h$ is faithfully flat if and only if $w$ is faithfully flat. By Lemma 1.17 this is the case if and only if $\varphi: G \longrightarrow H$ is surjective, so that $U=\mathrm{B}(\operatorname{Ker}(\varphi))$. The condition that $\mathrm{H}^{0}\left(\mathcal{O}_{\mathcal{Y}}\right)=k$ is equivalent to the condition that $v_{*} \mathcal{O}_{\mathcal{X}} \simeq \mathcal{O}_{\mathrm{B} H}$ and, by Lemma 1.22 , to the full faithfulness of the functor $\gamma^{*}: \operatorname{Vect}(\mathrm{B} H) \longrightarrow \operatorname{Vect}(\mathrm{B} G)$. This last condition is equivalent to the surjectivity of $\varphi: G \longrightarrow H$ when $H / \operatorname{Im}(\varphi)$ is affine (see [TZ1, Remark B.7]).

Lemma 2.10. Let $\mathcal{X}$ be an algebraic stack over a field $k, A$ a finite and local $k$-algebra with residue field $k$ and $\mathcal{F} \in \operatorname{Vect}(\mathcal{X} \times \operatorname{Spec} A)$. Let $\mathcal{X} \stackrel{i}{\longrightarrow} \mathcal{X} \times \operatorname{Spec} A \stackrel{f}{\longrightarrow} \mathcal{X}$ be the maps 
corresponding to $k \longrightarrow A \longrightarrow k$. Then there is a sequence of surjective maps of vector bundles

$$
f_{*} \mathcal{F}=\mathcal{G}_{N} \longrightarrow \mathcal{G}_{N-1} \longrightarrow \cdots \longrightarrow \mathcal{G}_{1} \longrightarrow \mathcal{G}_{0}=0
$$

such that $\operatorname{Ker}\left(\mathcal{G}_{l} \longrightarrow \mathcal{G}_{l-1}\right) \simeq i^{*} \mathcal{F}$ for all $1 \leqslant l \leqslant N$.

Proof. Consider a decomposition series of $A$-modules

$$
A=A_{N} \longrightarrow A_{N-1} \longrightarrow \cdots \longrightarrow A_{1} \longrightarrow A_{0}=0 ;
$$

the above maps are surjective with $\operatorname{Ker}\left(A_{l} \longrightarrow A_{l-1}\right) \simeq k$ as $A$-modules for all $1 \leqslant l \leqslant N$. Let $p: \mathcal{X} \times \operatorname{Spec} A \longrightarrow \operatorname{Spec} A$ be the projection; consider the functor

$$
\Psi=f_{*}\left(\mathcal{F} \otimes p^{*}(-)\right): \operatorname{Mod} A \longrightarrow \mathrm{QCoh}(\mathcal{X}) .
$$

Since $p$ is flat, $\mathcal{F}$ is a vector bundle, and as $f$ is affine the functor $\Psi$ is exact. Moreover $\Psi(A)=f_{*} \mathcal{F}$ and

$$
\Psi(k)=f_{*}\left(\mathcal{F} \otimes p^{*} k\right) \simeq f_{*}\left(\mathcal{F} \otimes i_{*} \mathcal{O}_{\mathcal{X}}\right) \simeq f_{*} i_{*} i^{*} \mathcal{F} \simeq i^{*} \mathcal{F} .
$$

Applying $\Psi$ to the above sequence of $A$-modules we find the desired sequence.

Lemma 2.11. Let $\mathcal{X}$ be a pseudo-proper and inflexible algebraic stack of finite type over a field $k$ of positive characteristic, such that $\operatorname{dim}_{k} \mathrm{H}^{1}(\mathcal{X}, E)<\infty$ for all vector bundles $E$ on $\mathcal{X}$. Let $f: \mathcal{Y} \longrightarrow \mathcal{X}$ be an essentially finite cover. Then for all maps $\phi: \mathcal{Y} \longrightarrow \Phi$ to a finite stack over $k$, and for all $W \in \operatorname{Vect}(\Phi)$, the vector bundle $f_{*} \phi^{*} W$ is essentially finite in $\operatorname{Vect}(\mathcal{X})$.

Proof. To avoid problems with different ranks, we first observe that the rank of $W$ can be assumed to be constant, for instance, by considering the connected components of $\Phi$. By Lemma 2.2 we have a Cartesian diagram

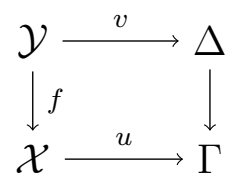

where $\Gamma$ is a finite gerbe. We will prove that there exists a surjective cover $s: \mathcal{X}^{\prime} \longrightarrow \mathcal{X}$ such that $s^{*} f_{*} \phi^{*} W$ is free on the connected components of $\mathcal{X}^{\prime}$; this will be done by only assuming that there is a Cartesian diagram as above with $\Gamma$ a finite gerbe and $f$ finite, but without requiring that $\mathcal{X}$ is inflexible, as this will allow us to replace $\mathcal{X}$ by any surjective cover of it. The lemma then follows from Theorem 1.15.

As mentioned above, if $s^{\prime}: \mathcal{T}^{\prime} \longrightarrow \mathcal{X}$ is a surjective cover we can always replace $\mathcal{X}$ by $\mathcal{T}^{\prime}$. Let $L / k$ be a finite field extension with a map $\operatorname{Spec} L \longrightarrow \mathcal{Y}$. The base change of Spec $L \longrightarrow \mathcal{Y} \longrightarrow \Delta \longrightarrow \Gamma$ along $\mathcal{X} \longrightarrow \Gamma$ is a surjective cover $\mathcal{Q} \longrightarrow \mathcal{X}$. Replacing $\mathcal{X}$ by $\mathcal{Q}$ we can assume that $\mathcal{X} \longrightarrow \Gamma$ factors as $\mathcal{X} \longrightarrow \operatorname{Spec} L \longrightarrow \Gamma$. This means that $\mathcal{Y} \stackrel{f}{\longrightarrow} \mathcal{X}$ is the projection $\mathcal{X} \times{ }_{L} A \longrightarrow \mathcal{X}$, where $A / L$ is a finite $L$-algebra. Since $\phi$ factors as $\mathcal{Y} \longrightarrow \Phi \times_{k} L \longrightarrow \Phi$ we can assume that $L=k$. Extending again $k$ we can further assume that $A$ is a product of local $k$-algebras with residue field $k$. Splitting $\mathcal{Y}$ according 
to the decomposition of $A$ we can assume that $A$ is also local. Let $i: \mathcal{X} \longrightarrow \mathcal{Y}=\mathcal{X} \times A$ be the inclusion corresponding to $A \longrightarrow k$.

Finally consider a surjective cover $T \longrightarrow \Phi$ from a finite scheme and the 2-Cartesian diagram

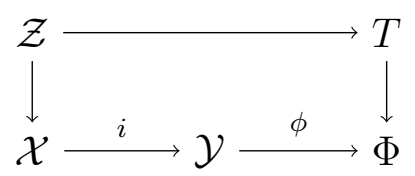

Replacing $\mathcal{X}$ by $\mathcal{Z}$ we can assume that $\phi \circ i: \mathcal{X} \longrightarrow \Phi$ factors through a finite scheme, which in particular implies that $i^{*} \phi^{*} W$ is free. Applying Lemma 2.10 to $\mathcal{F}=\phi^{*} W$ we obtain a sequence of surjective homomorphisms of quasi-coherent sheaves on $\mathcal{X}$

$$
f_{*}\left(\phi^{*} W\right)=\mathcal{G}_{N} \longrightarrow \mathcal{G}_{N-1} \longrightarrow \cdots \longrightarrow \mathcal{G}_{1} \longrightarrow \mathcal{G}_{0}=0
$$

with free kernels. The final cover trivializing $f_{*}\left(\phi^{*} W\right)$ exists due to Lemma 1.19.

Remark 2.12. Assume char $k=0$. Then the proof of Lemma 2.11 would not work anymore. The problem is that Lemma 1.19 holds only in positive characteristic:

Let $\mathcal{E} \operatorname{xt}(\mathcal{O}, \mathcal{O})$ be the stack over Aff $/ k$ which associates with each $T \in$ Aff $/ k$ the groupoid of extensions of the form

$$
0 \longrightarrow \mathcal{O}_{T} \longrightarrow E \longrightarrow \mathcal{O}_{T} \longrightarrow 0
$$

where $E$ is a quasi-coherent sheaf on $T$. A morphism between two extensions is given by a commutative diagram

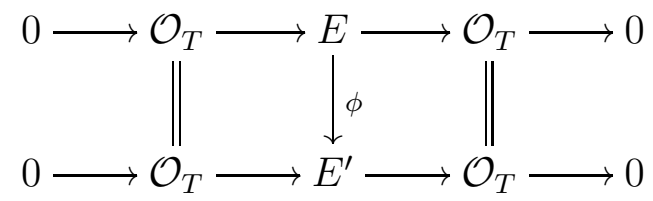

One checks readily that $\mathcal{E} x t(\mathcal{O}, \mathcal{O})$ is a trivial gerbe under $\mathbb{G}_{a}$, or in other words $\mathcal{E} x t(\mathcal{O}, \mathcal{O})=$ $\mathrm{B} \mathbb{G}_{a}$. Now let $\mathcal{X}=X$ be a proper geometrically connected reduced scheme over $k$ which admits a non-trivial extension of quasi-coherent sheaves:

$$
0 \longrightarrow \mathcal{O}_{X} \longrightarrow E \longrightarrow \mathcal{O}_{X} \longrightarrow 0
$$

If the conclusion of Lemma 1.19 was true in this case, then $E$ is essentially finite by Theorem 1.15. Let $\Gamma$ be the monodromy gerbe of $E$ in $\operatorname{EFin}(\operatorname{Vect}(X))$. Then we have the following commutative diagram

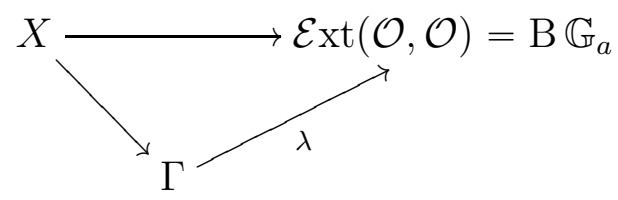

due to the full faithfulness of the pullback $\operatorname{Vect}(\Gamma) \longrightarrow \operatorname{EFin}(\operatorname{Vect}(X))$ and the fact that $\mathcal{O}_{X}$ and $E$ come from $\operatorname{Vect}(\Gamma)$. The map $\lambda$ is non-trivial because its composition with $X \longrightarrow \Gamma$ is non-trivial. But by [DG, Chapitre IV, $\S 2, n^{o} 1,1.1$, pp. 483] $\mathbb{G}_{a}$ has no non-trivial finite subgroup in characteristic 0 and therefore $\lambda$ has to be trivial, a contradiction. 
However, Lemma 2.11 still holds true in characteristic 0 when $\mathcal{Y}$ is inflexible. This is due to Lemma 2.13 and Proposition 2.14 below.

Lemma 2.13. Let $\mathcal{X}$ be a pseudo-proper and inflexible algebraic stack of finite type over $k$, and let $f: \mathcal{Y} \longrightarrow \mathcal{X}$ be an étale surjective cover. Then for all maps $\phi: \mathcal{Y} \longrightarrow \Phi$ to a finite (respectively, finite and étale) stack over $k$ and for all $W \in \operatorname{Vect}(\Phi)$, the vector bundle $f_{*}\left(\phi^{*} W\right)$ is essentially finite (respectively, essentially finite with étale monodromy gerbe) in $\operatorname{Vect}(\mathcal{X})$. In particular, $f$ is essentially finite.

Proof. There exists a Cartesian diagram

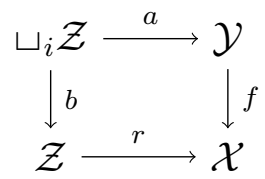

where $r: \mathcal{Z} \longrightarrow \mathcal{X}$ is an étale surjective cover. Since $a^{*} \phi^{*} W$ also comes from the finite stack $\Phi$, taking a finite atlas of $\Phi$ we can find a surjective cover $\lambda: \mathcal{U} \rightarrow \bigsqcup_{i} \mathcal{Z}$ which trivializes it. Denote by $\mathcal{U}_{i}$ the inverse image of the $i$-th piece of $\bigsqcup_{i} \mathcal{Z}$ under $\lambda$. Then $r^{*} f_{*} \phi^{*} W=b_{*} a^{*} \phi^{*} W$ is trivialized by the surjective cover $\mathcal{U}_{1} \times{ }_{\mathcal{Z}} \times \cdots \times{ }_{\mathcal{Z}} \mathcal{U}_{n} \longrightarrow \mathcal{Z}$. Thus, by Remark 1.16, $f_{*} \phi^{*} W$ is essentially finite and it has an étale monodromy gerbe if $\Phi$ is étale (so that $\lambda$ can also be chosen to be étale).

Proof of Theorem I. By Lemma 2.2 there are 2-Cartesian diagrams

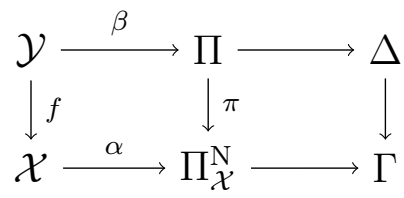

where $\Gamma$ is the monodromy gerbe of $f_{*} \mathcal{O}_{\mathcal{Y}} \in \operatorname{EFin}(\operatorname{Vect}(\mathcal{X}))$. It also follows from Lemma 2.2 that $\Pi$ is a gerbe if $\mathcal{Y}$ is inflexible.

For the converse assume that $\Pi$ is a gerbe. By Remark 1.23 and Lemma 2.7, the pullback functor $\beta^{*}: \operatorname{Vect}(\Pi) \longrightarrow \operatorname{Vect}(\mathcal{Y})$ is fully faithful with essential image the full subcategory $\mathcal{C}$ of $\operatorname{Vect}(\mathcal{Y})$ of vector bundles $V$ such that $f_{*} V \in \operatorname{EFin}(\operatorname{Vect}(\mathcal{X}))$. Since $\Pi \longrightarrow \Pi_{\mathcal{X}}^{\mathrm{N}}$ is faithful the gerbe $\Pi$ is profinite, so we have $\mathcal{C} \subseteq \operatorname{EFin}(\operatorname{Vect}(\mathcal{Y}))$. We will show that this is an equality and that $\mathcal{Y}$ is inflexible. This would immediately imply that $\mathcal{Y} \longrightarrow \Pi$ is the Nori fundamental gerbe. Let $\phi: \mathcal{Y} \longrightarrow \Phi$ be a map to a finite stack. Using Lemma 2.13 (notice that since $\Pi$ is gerbe, $\Delta$ is also a gerbe, so if char $k=0$ then $\Delta \longrightarrow \Gamma$ and $f: \mathcal{Y} \longrightarrow \mathcal{X}$ are étale covers) and Lemma 2.11 it follows that $\phi^{*} W \in \mathcal{C}$ for all $W \in \operatorname{Vect}(\Phi)$. Thus the pullback by $\phi: \mathcal{Y} \longrightarrow \Phi$ has a factorization

$$
\operatorname{Vect}(\Phi) \longrightarrow \operatorname{Vect}(\Pi) \simeq \mathcal{C} \subseteq \operatorname{Vect}(\mathcal{Y})
$$

By Remark 1.20 one gets a factorization $\mathcal{Y} \longrightarrow \Pi \longrightarrow \Phi$ as required. This shows that $\mathcal{Y} \longrightarrow \Pi$ is a Nori fundamental gerbe and, in particular, $\mathcal{Y}$ is inflexible. Since $\mathcal{Y}$ is pseudoproper, all essentially finite vector bundles on $\mathcal{Y}$ are pullbacks from some finite gerbe. Thus the above factorization also implies the equality $\mathcal{C}=\operatorname{EFin}(\operatorname{Vect}(\mathcal{Y}))$. 
Notice that if $\mathcal{Y}$ is inflexible then one always has $\mathrm{H}^{0}\left(\mathcal{O}_{\mathcal{Y}}\right)=k$ (see Remark 1.8).

The étale case. Assume that $f$ is étale and that $\mathrm{H}^{0}\left(\mathcal{O}_{\mathcal{Y}}\right)=k$. By Lemma 2.7 we have $\mathrm{H}^{0}\left(\mathcal{O}_{\Delta}\right)=k$. In particular, $\Delta$ is geometrically connected. Since $\Delta \longrightarrow \Gamma$ is étale, it follows that $\Delta$ is also geometrically reduced. Using [BV, Proposition 4.3] we conclude that $\Delta$ and therefore $\Pi$ are gerbes. Thus $\mathcal{Y}$ is inflexible. By Lemma 2.13 we see that $\Gamma$ is étale. In particular there are 2-Cartesian diagrams

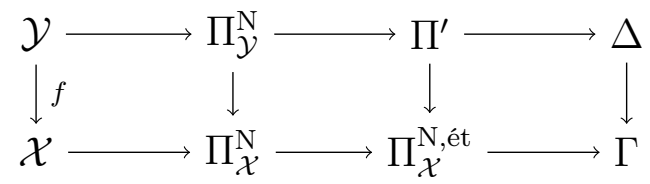

We want to show that $\Pi^{\prime}=\Pi_{\mathcal{Y}}^{\mathrm{N} \text {,ét }}$. By Lemma 2.7 the pullback $\operatorname{Vect}\left(\Pi^{\prime}\right) \longrightarrow \operatorname{Vect}(\mathcal{Y})$ is fully faithful and its essential image $\mathcal{C}$ consists of vector bundles $V \in \operatorname{Vect}(\mathcal{Y})$ such that $f_{*} V$ comes from $\Pi_{\mathcal{X}}^{\mathrm{N} \text { ét }}$. One must show that the essential image $\mathcal{D}$ of the fully faithful map $\operatorname{Vect}\left(\Pi_{\mathcal{Y}}^{\mathrm{N}, \text { ét }}\right) \longrightarrow \operatorname{Vect}(\mathcal{Y})$ coincides with $\mathcal{C}$. The vector bundles in $\mathcal{D}$ are the essentially finite vector bundles with étale monodromy gerbes. Since the map $\Pi^{\prime} \longrightarrow \Pi_{\mathcal{X}}^{\mathrm{N} \text {,ét }}$ is faithful, it follows that $\Pi^{\prime}$ is proétale, so that $\mathcal{C} \subseteq \mathcal{D}$. The opposite inclusion instead follows from Lemma 2.13.

The torsor case. Assume that $f$ is a torsor for a finite group scheme $G$. Since the regular representation $k[G]$ generates $\operatorname{Rep}(G)$ as $k$-Tannakian category, there are 2-Cartesian diagrams

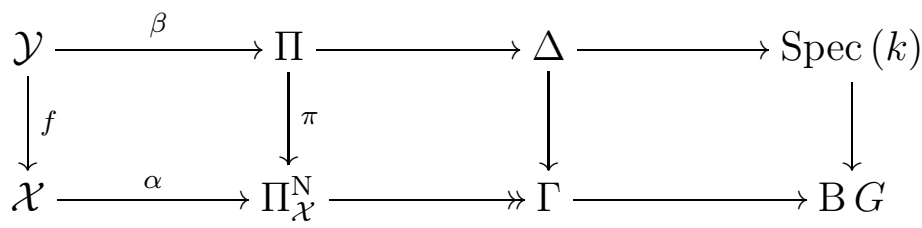

and the map $\Gamma \longrightarrow \mathrm{B} G$ is faithful and, by [TZ1, Remark B.7], affine. In particular $\Delta$ is a finite scheme, and by Lemma 2.7 we can conclude that $\Delta=\operatorname{Spec}\left(\mathrm{H}^{0}\left(\mathcal{O}_{\mathcal{Y}}\right)\right)$. Now the theorem follows because $\mathcal{Y}$ is inflexible if and only if $\Delta$ is a gerbe.

Proof of Corollary I. The Nori gerbe of $\mathcal{X}$ is $\Pi_{\mathcal{X}}^{\mathrm{N}}=\mathrm{B} \pi^{\mathrm{N}}(\mathcal{X}, x)$, and $\mathcal{X} \longrightarrow \mathrm{B} \pi^{\mathrm{N}}(\mathcal{X}, x)$ maps $x$ to the trivial torsor. If $(\mathcal{Y}, y) \stackrel{f}{\longrightarrow}(\mathcal{X}, x)$ is an essentially finite cover, then the extension $\Pi \longrightarrow \Pi_{\mathcal{X}}^{\mathrm{N}}$ defined in Theorem I is described by the 2-Cartesian diagrams

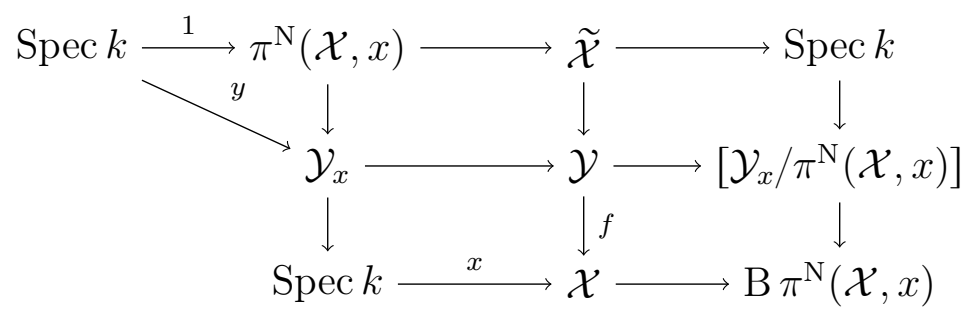

that is $\Pi=\left[\mathcal{Y}_{x} / \pi^{\mathrm{N}}(\mathcal{X}, x)\right]$. Again by Theorem I we know that $\mathcal{Y}$ is inflexible if and only if $\Pi$ is a gerbe. On the other hand, the following three conditions are equivalent: 
- $\Pi$ is a gerbe,

- Spec $k \longrightarrow \Pi$ is faithfully flat, and

- the orbit map $\pi^{\mathrm{N}}(\mathcal{X}, x) \longrightarrow \mathcal{Y}_{x}$ of $y$ is faithfully flat.

When these equivalent conditions hold, by Theorem I we have $\Pi=\mathrm{B} \pi^{\mathrm{N}}(\mathcal{Y}, y)$ and $\mathcal{Y}_{x} \simeq$ $\pi^{\mathrm{N}}(\mathcal{X}, x) / \pi^{\mathrm{N}}(\mathcal{Y}, y)$. The equivalence of categories in the statement follows easily from Proposition 2.1.

Let $f:(\mathcal{Y}, y) \longrightarrow(\mathcal{X}, x)$ be an essentially finite cover with $\mathcal{Y}$ inflexible. If $f$ is a torsor under a group $G$, using Lemma 1.17 (1) the last diagram in Theorem I tells that $\pi^{\mathrm{N}}(\mathcal{Y}, y)$ is normal in $\pi^{\mathrm{N}}(\mathcal{X}, x)$ with quotient $G$. Conversely, if $\pi^{\mathrm{N}}(\mathcal{Y}, y)$ is normal in $\pi^{\mathrm{N}}(\mathcal{X}, x)$ with quotient $G$, then using again Lemma $1.17(1), \mathrm{B} \pi^{\mathrm{N}}(\mathcal{Y}, y) \longrightarrow \mathrm{B} \pi^{\mathrm{N}}(\mathcal{X}, x)$ and its base change $f: \mathcal{Y} \longrightarrow \mathcal{X}$ is torsor under $G$.

For the last claim, considering the above Cartesian diagrams we see that the following three are equivalent:

- $f$ is étale,

- $\Pi=\mathrm{B} \pi^{\mathrm{N}}(\mathcal{Y}, y) \longrightarrow \mathrm{B} \pi^{\mathrm{N}}(\mathcal{X}, x)$ is étale,

- $\mathcal{Y}_{x} \simeq \pi^{\mathrm{N}}(\mathcal{X}, x) / \pi^{\mathrm{N}}(\mathcal{Y}, y) \longrightarrow$ Spec $k$ is étale.

This completes the proof.

Proposition 2.14. Let $\mathcal{X}$ be a pseudo-proper and inflexible fibered category over $k$ and $f: \mathcal{Y} \longrightarrow \mathcal{X}$ be a cover. The following are equivalent:

(1) $\mathcal{Y}$ is inflexible, $f$ is essentially finite and $f_{*} \mathcal{O}_{\mathcal{Y}}$ has étale monodromy gerbe in $\operatorname{EFin}(\operatorname{Vect}(\mathcal{X}))$

(2) $f$ is étale and $\mathrm{H}^{0}\left(\mathcal{O}_{\mathcal{Y}}\right)=k$.

Proof. The implication (2) $\Longrightarrow$ (1) follows from Lemma 2.13 and Theorem I. For the converse, let $\Delta \longrightarrow \Gamma$ be as in Lemma 2.2. Since $\mathcal{Y}$ is inflexible it follows that $\Delta$ is a gerbe. Moreover by hypothesis $\Gamma$ is étale. Thus $\Delta$ is étale too because $\Delta \longrightarrow \Gamma$ is faithful. By base change it follows that $f$ is étale.

\section{TOWERS OF TORSORS AND THEIR GalOIS ClOSURES}

Let $k$ be a base field and $G$ and $H$ be finite group schemes over $k$. In this section we introduce the notion of tower of torsors and Galois closure of a tower of torsors. At the end of the section Theorems 3.9 and III will be proved.

Definition 3.1. A $(G, H)$-tower of torsors over a fibered category $\mathcal{X}$ over $k$ is a sequence of map of fibered categories $\mathcal{Z} \stackrel{h}{\longrightarrow} \mathcal{Y} \stackrel{g}{\longrightarrow} \mathcal{X}$ where $g$ is a $G$-torsor and $h$ is an $H$-torsor. When $G, H$ are clear from the context we will just talk about a tower of torsors. The $(G, H)$-tower is called pointed over $k$ if $\mathcal{Z} \longrightarrow \mathcal{X}$ (and therefore $\mathcal{Y} \longrightarrow \mathcal{X}$ ) is a pointed cover over $k$. 
We define the stack $\mathrm{B}(G, H)$ as the stack over Aff $/ k$ whose section over an affine scheme $U$ is the groupoid of $(G, H)$-tower of torsors over $U$. A morphism between two $(G, H)$ towers $\mathcal{Z} \stackrel{h}{\longrightarrow} \mathcal{Y} \stackrel{g}{\longrightarrow} \mathcal{X}$ and $\mathcal{Z} \stackrel{h^{\prime}}{\longrightarrow} \mathcal{Y}^{\prime} \stackrel{g^{\prime}}{\longrightarrow} \mathcal{X}$ is a commutative diagram

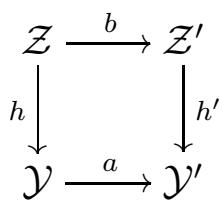

of $\mathcal{X}$-maps such that $a$ is $G$-equivariant and $\mathcal{Z} \longrightarrow \mathcal{Z}^{\prime} \times \mathcal{Y}^{\prime} \mathcal{Y}$ is $H$-equivariant. Clearly, $a, b$ are isomorphisms so that the stack $\mathrm{B}(G, H)$ is a groupoid.

Let us start with a preliminary remark:

Remark 3.2. A tower of torsors over a fibered category $\mathcal{X}$ is the same as a map $\mathcal{X} \longrightarrow$ $\mathrm{B}(G, H)$. Moreover $\mathrm{B}(G, H)$ has a universal tower given by

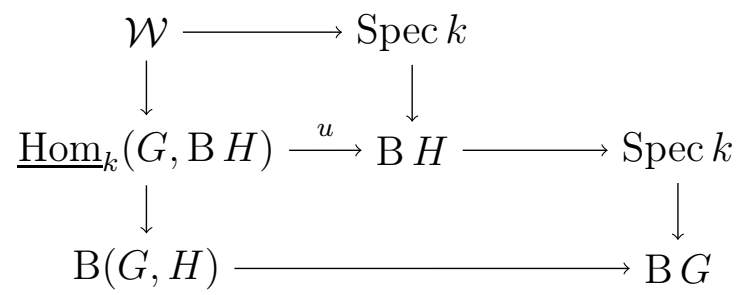

where $u$ is the restriction along $1: \operatorname{Spec} k \longrightarrow G$. The pullback of the above tower along a map $\mathcal{X} \longrightarrow \mathrm{B}(G, H)$ yields exactly the tower encoded in the map $\mathcal{X} \longrightarrow \mathrm{B}(G, H)$.

Proposition 3.3. The stack $\mathrm{B}(G, H)$ is algebraic, locally of finite type over $k$ and has affine diagonal.

Proof. The stack $\mathrm{B}(G, H)$ is algebraic and locally of finite type over the field $k$ because $\underline{\operatorname{Hom}}_{k}(G, \mathrm{~B} H)$ is so by [HR, Theorem 3] and the fact that there is a finite and flat map

$$
\underline{\operatorname{Hom}}_{k}(G, \mathrm{~B} H) \longrightarrow \mathrm{B}(G, H)
$$

by Remark 3.2.

Now let $P_{i} \longrightarrow P_{i}^{\prime} \longrightarrow T, i=1,2$, be two towers $\xi_{i} \in \mathrm{B}(G, H)(T)$ for some affine scheme $T$, and set $I=\underline{\operatorname{IsO}}_{\mathrm{B}(G, H)}\left(\xi_{1}, \xi_{2}\right)$. We need to show that $I$ is affine. We denote by $(-)_{T}$ the base change to $T$. Base changing along $P_{1}^{\prime} \times_{T} P_{2}^{\prime} \longrightarrow T$ allows us to assume that $P_{i}^{\prime}=G_{T}$. In particular, there is a map $a: I \longrightarrow G_{T}=\underline{\mathrm{Aut}}_{T}^{G}\left(G_{T}\right)$ and we want to show that it is affine. If $W$ is the fiber of $a$ along a map $T \stackrel{g}{\longrightarrow} G_{T}$, it is enough to show that $W$ is affine. Let $\bar{P}_{2} \longrightarrow G_{T}$ be the $H$-torsor base change of $P_{2} \longrightarrow G_{T}$ along the multiplication $G_{T} \longrightarrow G_{T}$ by $g$ and set $J=\underline{\operatorname{Iso}}_{G_{T}}^{H}\left(P_{1}, \bar{P}_{2}\right) \longrightarrow G_{T}$. It is easy to see that $W=W_{G_{T}}(J)$, where $W_{G_{T}}(J)$ is the Weil restriction of $J$ along $G_{T} \longrightarrow T$. The scheme $J$ is affine and finitely presented over $G_{T}$. Since $G_{T} \longrightarrow T$ is a cover using a presentation of $J$ it is easy to write $W$ as a closed subscheme of an affine space over $T$. 
Lemma 3.4. Let $\mathcal{X}$ be a pseudo-proper and inflexible algebraic stack of finite type over $k$, and let $\mathcal{Z} \stackrel{h}{\longrightarrow} \mathcal{Y} \stackrel{g}{\longrightarrow} \mathcal{X}$ be a $(G, H)$-tower of torsors. If char $k>0$ assume that either $g$ or $h$ is étale or $\operatorname{dim}_{k} \mathrm{H}^{1}(\mathcal{X}, E)<\infty$ for every vector bundle $E$ on $\mathcal{X}$. Then $g \circ h$ is an essentially finite cover.

Proof. Consider the morphism $v: \mathcal{Y} \longrightarrow$ B $H$ corresponding to the $H$-torsor $h: \mathcal{Z} \longrightarrow \mathcal{Y}$. One knows that $h_{*} \mathcal{O}_{\mathcal{Z}}=v^{*}(k[H])$, where $k[H]$ denotes the regular representation. One then concludes the proof by Lemma 2.11 and Theorem 3.9.

Lemma 3.5. Let $P \longrightarrow G \longrightarrow$ Spec $k$ be a rational point $\xi$ of $\mathrm{B}(G, H)$ in which $G \longrightarrow$ Spec $k$ is the trivial $G$-torsor, and let $p \in P(k)$ be a rational point mapping to $1 \in G(k)$, and let $Q_{\xi}=\underline{\operatorname{Aut}}_{\mathrm{B}(G, H)}(\xi)$. Then $Q_{\xi}$ is an affine group scheme of finite type over $k$, and there are exact sequences

$$
0 \longrightarrow W_{G / k}\left(\underline{\operatorname{Aut}}_{G}^{H}(P)\right) \longrightarrow Q_{\xi} \stackrel{\alpha}{\longrightarrow} G, \quad 0 \longrightarrow W^{\prime} \longrightarrow W_{G / k}\left(\underline{\mathrm{Aut}}_{G}^{H}(P)\right) \stackrel{\beta}{\longrightarrow} H
$$

where $\alpha$ forgets the automorphism of $P, W_{G / k}$ denotes the Weil restriction and $\beta$ is the evaluation at $1:$ Spec $k \longrightarrow G$.

There is a fully faithful map $\mathrm{B} Q_{\xi} \longrightarrow \mathrm{B}(G, H)$ sending the trivial torsor to $\xi$ and whose image consists of the substack of towers locally isomorphic to $\xi$.

Proof. The scheme $Q_{\xi}$ is affine of finite type by Proposition 3.3. The first sequence is clear. The map $\beta$ is well-defined because the point $p \in P(k)$ gives an $H$-equivariant isomorphism between $H$ and the base change of $P \longrightarrow G$ along the identity: the base change of $\underline{\mathrm{Aut}}_{G}^{H}(P) \longrightarrow G$ along the identity is $\underline{\mathrm{Aut}}_{k}^{H}(H)=H$. The last claim is standard.

More can be said in the case of the trivial tower.

Lemma 3.6. Let $Q$ be the sheaf of automorphisms of the trivial tower

$$
G \times H \longrightarrow G \longrightarrow \operatorname{Spec} k
$$

in $\mathrm{B}(G, H)$. Then $Q$ is an affine group scheme of finite type,

$$
Q=W_{G}(H) \ltimes G
$$

where $W_{G}(H)$ is the Weil restriction of the group scheme $H \times{ }_{k} G$ over $G$ along $G \longrightarrow$ Spec $k$ with $G$ acting on $W_{G}(H)$ via automorphisms of the base. Evaluation at $1 \in G$ yields a map $W_{G}(H) \longrightarrow H$ and, if $W^{\prime}$ is the kernel, then

$$
W_{G}(H)=W^{\prime} \ltimes H .
$$

The fully faithful map $\mathrm{B} Q \longrightarrow \mathrm{B}(G, H)$ of Lemma 3.5 corresponds to the pointed tower of Nori-reduced torsors

$$
\mathrm{B} W^{\prime} \longrightarrow \mathrm{B} W_{G}(H) \longrightarrow \mathrm{B} Q .
$$

If $G$ or $H$ is étale over $k$ then $Q$ is a finite group scheme. 
Proof. Consider the maps $\alpha$ and $\beta$ defined in Lemma 3.5. We have $P=G \times H$, so that

$$
\underline{\operatorname{Aut}}_{G}^{H}(P)=H \times G \longrightarrow G .
$$

Moreover it is easy to see that both $\alpha$ and $\beta$ are surjective. The map

$$
G \longrightarrow Q, \quad g \longmapsto\left(t_{g} \times \operatorname{id}_{H}, t_{g}\right)
$$

where $t_{g}$ is the multiplication by $g$, produces the first decomposition. The map $H(T) \longrightarrow$ $H(G \times T)=W_{G}(H)(T)$ produces the second decomposition.

The claim about the tower of B $Q$ is an easy consequence of Lemma 1.17.

Now assume that $G$ or $H$ are étale. We can also assume that $k$ is algebraically closed, so that $G$ or $H$ are constant. If $G$ is constant then $W_{G}(H)=H^{\# G}$ is a finite scheme and therefore $Q$ is finite. Thus assume $H$ constant. The map $G_{\text {red }} \longrightarrow G$, where $(-)_{\text {red }}$ denotes the reduction, is a nilpotent closed immersion and, since $H$ is étale, it follows that the map $W_{G}(H) \longrightarrow W_{G_{\text {red }}}(H)$ is an isomorphism. But $G_{\text {red }}$ is equal to the étale quotient $G_{\text {ét }}$ of $G$ (because $k$ is perfect) and again we conclude that $W_{G_{\text {red }}}(H)$ is finite.

Proposition 3.7. If $G$ or $H$ is étale then the map $\mathrm{B} Q \longrightarrow \mathrm{B}(H, G)$ is an equivalence, where $Q$ is the sheaf of automorphisms of the trivial tower $G \times H \longrightarrow G \longrightarrow$ Spec $k$ in $\mathrm{B}(G, H)$. In particular, $\mathrm{B}(G, H)$ is a finite neutral gerbe over $k$.

Proof. In view of Lemma 3.6 it suffices to show that any tower is fpqc locally trivial.

Let $P \longrightarrow P^{\prime} \longrightarrow U$ be a tower over an affine scheme. Using base changing along $P \longrightarrow U$ we may assume this map has a section, so that, in particular, $P^{\prime}=U \times G$. We can also assume that $k$ is algebraically closed, so that $G$ or $H$ is constant. If $G$ is constant, $P \longrightarrow U \times G$ is given by a number \#G of $H$-torsors over $U$ and, trivializing those torsors, one gets a trivialization of $P \longrightarrow U \times G$. Now consider $H$ to be étale. Since $P \longrightarrow U \times G$ is étale and $U \times G_{\text {red }} \longrightarrow U \times G$ is a nilpotent closed immersion we conclude that $P \longrightarrow U \times G$ has a section if and only if its restriction to $U \times G_{\text {red }}$ is trivial. Thus we reduce to the known case where $G=G_{\text {red }}=G_{\text {ét }}$ is étale.

We now move to the problem of finding a Galois closure for a given tower of torsors.

Definition 3.8. Let $\mathcal{X}$ be a fibered category, and let $\mathcal{Z} \longrightarrow \mathcal{Y} \longrightarrow \mathcal{X}$ be a $(G, H)$-tower of torsors. A Galois envelope for the $(G, H)$-tower consists of the following data:

- a finite group scheme $\mathcal{G}$ with homomorphisms of group schemes $\alpha: \mathcal{G} \longrightarrow G$ and $\operatorname{Ker}(\alpha) \longrightarrow H$

- a $\mathcal{G}$-torsor $\mathcal{P} \longrightarrow \mathcal{X}$ together with a factorization $\mathcal{P} \longrightarrow \mathcal{Z}$ such that $\mathcal{P} \longrightarrow \mathcal{Y}$ is $\mathcal{G}$-equivariant and $\mathcal{P} \longrightarrow \mathcal{Z}$ is $\operatorname{Ker}(\alpha)$-equivariant.

We say that the Galois envelope is Nori-reduced if the $\mathcal{G}$-torsor $\mathcal{P} \longrightarrow \mathcal{X}$ is Nori-reduced. If $\mathcal{Z} \longrightarrow \mathcal{X}$ is an essentially finite cover, and if the Galois envelope $\mathcal{P} \longrightarrow \mathcal{X}$ coincides with the Galois closure of the essentially finite cover, then we call $\mathcal{P} \longrightarrow \mathcal{X}$ the Galois closure for the $(G, H)$-tower.

Now we get a beautiful Galois envelope for the case when either $G$ or $H$ is étale:

Theorem 3.9. If $\mathcal{X}$ is a category fibered over $k$ and $G$ or $H$ is étale then 
(1) every $(G, H)$-tower of torsors $\mathcal{Z} \stackrel{h}{\longrightarrow} \mathcal{Y} \stackrel{g}{\longrightarrow} \mathcal{X}$ is an essentially finite cover and,

(2) it admits a canonical Galois envelope $\eta: \mathcal{P} \longrightarrow \mathcal{X}, \lambda: \mathcal{P} \longrightarrow \mathcal{Z}$, where $\eta$ is a torsor under the finite group scheme $Q$ representing the sheaf of automorphisms of the trivial tower $G \times H \longrightarrow G \longrightarrow$ Spec $k$;

(3) the map $\lambda$ is a torsor under a finite subgroup scheme of $Q$;

(4) if $\mathcal{X}$ is pseudo-proper and inflexible, then $\eta: \mathcal{P} \longrightarrow \mathcal{X}$ is the Galois closure for the $(G, H)$-tower if and only if the corresponding map $\mathcal{X} \longrightarrow \mathrm{B}(G, H)=\mathrm{B} Q$ is Nori-reduced.

Proof. By Lemma 3.6 and Proposition 3.7 we have $\mathrm{B}(G, H)=\mathrm{B} Q$, with universal tower $\mathrm{B} W^{\prime} \stackrel{b}{\longrightarrow} \mathrm{B} W_{G}(H) \stackrel{a}{\longrightarrow} \mathrm{B} Q$. The $Q$-torsor Spec $k \longrightarrow \mathrm{B} Q$ with splitting $\operatorname{Spec} k \longrightarrow$ $\mathrm{B} W^{\prime}$ gives a Galois closure of the universal tower. Now for any $(G, H)$-tower $\mathcal{X} \longrightarrow$ $\mathrm{B}(G, H)=\mathrm{B} Q$, the Galois envelope $\mathcal{P} \longrightarrow \mathcal{X}$ is just the pullback of Spec $k \longrightarrow \mathrm{B} Q$ along $\mathcal{X} \longrightarrow \mathrm{B}(G, H)=\mathrm{B} Q$. Since $(g \circ h)_{*} \mathcal{O}_{\mathcal{Z}}$ is the pullback of $(a \circ b)_{*} \mathcal{O}_{\mathrm{B} W^{\prime}}$ along $\mathcal{X} \longrightarrow \mathrm{B}(G, H)=\mathrm{B} Q$, it is essentially finite. This finishes (1), (2), (3).

Let $\left\langle(a \circ b)_{*} \mathcal{O}_{\mathrm{B} W^{\prime}}\right\rangle$ be the full Tannakian subcategory of $\mathrm{B} Q$ generated by $(a \circ b)_{*} \mathcal{O}_{\mathrm{B} W^{\prime}}$ with Galois group $Q^{\prime}$. Then the surjection $Q \rightarrow G$ factors as $Q \rightarrow Q^{\prime} \rightarrow G$. Let $W$ be the inverse image of $Q^{\prime}$ under $W_{G}(H) \hookrightarrow Q$. Then we have a Cartesian diagram

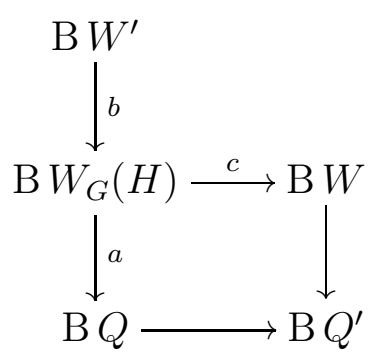

By Lemma 2.7 we see that $b_{*} \mathcal{O}_{\mathrm{B} W^{\prime}}$ is a pullback of a vector bundle on $\mathrm{B} W$. By the same argument of Lemma 2.2 we can complete the arrows $b, c$ to a Cartesian diagram where the northeastern Vertex is a gerbe. This implies that $\operatorname{Ker}\left(W_{G}(H)\right) \stackrel{c}{\longrightarrow} W$ is contained in $W^{\prime}$, so $\mathrm{B} W_{G}(H) \longrightarrow \mathrm{B} H$ factors through $c$. In this way we obtain a tower for the stack $\mathrm{B} Q^{\prime}$. By the universal property of the tower on $\mathrm{B} Q$ we conclude $Q=Q^{\prime}$. Thus the regular representation of $Q$ (i.e. the pushforward of $\mathcal{O}_{\text {Spec } k}$ along Spec $k \longrightarrow \mathrm{B} Q$ ) is a subquotient of $f\left((a \circ b)_{*} \mathcal{O}_{\mathrm{B} W^{\prime}},(a \circ b)_{*} \mathcal{O}_{\mathrm{B} W^{\prime}}\right)$ for some $f(X, Y) \in \mathbb{N}[X, Y]$. Conversely, $(a \circ b)_{*} \mathcal{O}_{\mathrm{B} W^{\prime}}$ is obviously a subobject of the regular representation of $Q$.

If $\mathcal{X}$ pseudo-proper and inflexible, then the above shows that the Tannakian subcategory of $\operatorname{EFin}(\mathcal{X})$ generated by $\eta_{*} \mathcal{O}_{\mathcal{P}}$ is the same as the essential image of the pullback functor $\operatorname{Vect}(\mathrm{B} Q) \longrightarrow \operatorname{EFin}(\mathcal{X})$ (which is the Tannakian subcategory generated by $(g \circ h)_{*} \mathcal{O}_{\mathcal{Z}}$ ). Thus $\mathcal{P} \longrightarrow \mathcal{X}$ is identified with the Galois closure $\mathcal{P}_{f}$ of $\mathcal{Z} \stackrel{f:=g \circ h}{\longrightarrow} \mathcal{X}$ constructed in Theorem II if and only if $\mathcal{X} \longrightarrow \mathrm{B} Q$ is Nori-reduced.

The following lemma shows that a pointed tower $\mathcal{Z} \longrightarrow \mathcal{Y} \longrightarrow \mathcal{X}$ has a Galois closure. 
Theorem 3.10. Let $\mathcal{X}$ be a pseudo-proper and inflexible algebraic stack of finite type over $k$ and $\mathcal{Z} \stackrel{h}{\rightarrow} \mathcal{Y} \stackrel{g}{\rightarrow} \mathcal{X}$ a pointed tower of torsors. If char $k>0$, assume that either $g$ is étale or $\operatorname{dim}_{k} \mathrm{H}^{1}(\mathcal{X}, E)<\infty$ for every vector bundle $E$ on $\mathcal{X}$. Then the following hold:

(1) $f: \mathcal{Z} \longrightarrow \mathcal{X}$ is essentially finite,

(2) the map $\omega: \mathcal{X} \longrightarrow \mathrm{B}(G, H)$ corresponding to the tower factors through the monodromy gerbe $\mathcal{X} \stackrel{\phi}{\longrightarrow} \Gamma=\mathrm{B} G_{f}$ of $f_{*} \mathcal{O}_{\mathcal{Z}}$ in $\operatorname{EFin}(\operatorname{Vect}(\mathcal{X}))$ and

(3) the $G_{f}$-torsor $\mathcal{P}_{f} \longrightarrow \mathcal{X}$ and the factorization $\mathcal{P}_{f} \longrightarrow \mathcal{Z}$ introduced in Theorem II define a Galois closure for the $(G, H)$-tower $\mathcal{Z} \longrightarrow \mathcal{Y} \longrightarrow \mathcal{X}$.

Moreover, the group scheme $G_{f}$ is a finite subgroup of the affine and finite type k-group scheme $\underline{\operatorname{Aut}}_{\mathrm{B}(G, H)}(\omega(x))$, where $x$ is the given rational point of $\mathcal{X}$.

Proof. The cover $f: \mathcal{Z} \longrightarrow \mathcal{X}$ is essentially finite by Lemma 3.4. We want to extend the given tower along $\mathcal{X} \stackrel{\phi}{\longrightarrow} \mathrm{B} G_{f}$ as in the diagram below. Using the notation of Theorem II (but the map $f: \mathcal{Y} \longrightarrow \mathcal{X}$ there is replaced by $f: \mathcal{Z} \longrightarrow \mathcal{X}$ in our case), according to Lemma 2.9 there exists a morphism of group schemes $G_{f} \longrightarrow G$ inducing the morphism $\mathcal{P}_{f} \longrightarrow \mathcal{Y}$. By Lemma 2.2 the cover $\mathcal{Z} \longrightarrow \mathcal{X}$ extends to a cover $\Delta \longrightarrow \mathrm{B} G_{f}$. Let $U:=\mathrm{B} G_{f} \times{ }_{\mathrm{B} G}$ Spec $k$. We have the following Cartesian diagrams:

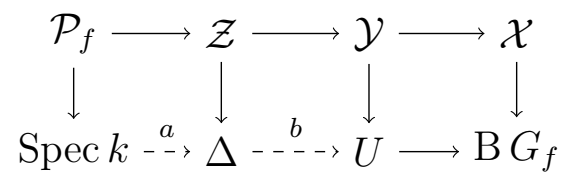

where the dashed arrows come from the existing maps $\mathcal{P}_{f} \longrightarrow \mathcal{Z}$ (for $a$ ) and $\mathcal{Z} \longrightarrow \mathcal{Y}$ (for $b)$ and the full faithfulness of $\operatorname{Vect}\left(\mathrm{B} G_{f}\right) \longrightarrow \operatorname{Vect}(\mathcal{X})$. We must equip $b: \Delta \longrightarrow U$ with a compatible structure of $H$-torsor. Notice that, by Lemma 2.7, a vector bundle on $\mathcal{Y}$ whose pullback to $\mathcal{Z}$ is free (and thus comes from a vector bundle on $\Delta$ ) comes from a vector bundle on $U$. Moreover Lemma 2.7 also tells us that $\operatorname{Vect}(U) \longrightarrow \operatorname{Vect}(\mathcal{Y})$ is fully faithful. This shows that we get a factorization

$$
\operatorname{Vect}(\mathrm{B} H) \longrightarrow \operatorname{Vect}(U) \longrightarrow \operatorname{Vect}(\mathcal{Y})
$$

and, by Tannakian duality, a factorization $\mathcal{Y} \longrightarrow U \longrightarrow \mathrm{B} H$. This determines an $H$-torsor $\Delta^{\prime} \longrightarrow U$ extending $\mathcal{Z} \longrightarrow \mathcal{Y}$. Since $\operatorname{Vect}(U) \longrightarrow \operatorname{Vect}(\mathcal{Y})$ is fully faithful one concludes that $\Delta^{\prime} \simeq \Delta$ over $U$.

Let $K$ be the kernel of $G_{f} \longrightarrow G$. The map Spec $k \longrightarrow U$ factors through a closed immersion $\mathrm{B} K \longrightarrow U$ and the composition $\mathrm{B} K \longrightarrow U \longrightarrow \mathrm{B} H$ preserves trivial torsors, meaning it is induced by a homomorphism $K \longrightarrow H$. It is easy to show that all the data constructed define a Galois envelope of the original tower with Galois group $\mathcal{G}=G_{f}$.

For the last claim, set $\xi=\omega(x)$ and $Q=\underline{\operatorname{Aut}}_{\mathrm{B}(G, H)}(\xi)$. By hypothesis the tower $\xi$ is pointed and therefore, by Lemma 3.5, the group scheme $Q$ is affine and of finite type and there is a fully faithful map $\mathrm{B} Q \longrightarrow \mathrm{B}(G, H)$ whose essential image is the full substack of $\mathrm{B}(G, H)$ of towers which are fpqc locally isomorphic to $\xi$. Since $\omega$ factors as $\mathcal{X} \longrightarrow \mathrm{B} G_{f} \longrightarrow \mathrm{B}(G, H)$, all objects in the image of $\mathrm{B} G_{f} \longrightarrow \mathrm{B}(G, H)$ are locally 
isomorphic to $\xi$. Thus the previous morphism factors through $\mathrm{B} G_{f} \longrightarrow \mathrm{B} Q$. This map

preserves trivial torsors and it is therefore induced by a map $G_{f} \stackrel{q}{\longrightarrow} Q$. Let $\mathcal{G}$ be the image of $q$. The factorization

$$
\mathcal{X} \longrightarrow \mathrm{B} G_{f} \longrightarrow \mathrm{B} \mathcal{G} \longrightarrow \mathrm{B} Q \subseteq \mathrm{B}(G, H)
$$

tells us that the tower over $\mathcal{X}$ extends to a tower over B $\mathcal{G}$ and therefore $f_{*} \mathcal{O}_{\mathcal{Z}}$ comes from a vector bundle on $\mathrm{B} \mathcal{G}$. But B $G_{f}$ is exactly the monodromy gerbe of $f_{*} \mathcal{O}_{\mathcal{Z}}$ in $\operatorname{EFin}(\operatorname{Vect}(\mathcal{X}))$, which implies that $G_{f}=\mathcal{G} \subseteq Q$.

Proof of Theorem III. The closure we consider is the one in Theorem 3.10. Statement (1) follows by applying Theorem I and lemma 2.9 to both $\mathcal{X}$ and $\mathcal{Y}$ as bases. Statement (2) follows from the last statement of Theorem 3.10.

\section{The S-Fundamental GERBE OF ESSENTIALly Finite COVERS}

The aim of this section is to prove Theorem IV. We start by introducing the S-fundamental gerbe, which generalizes the notion of S-fundamental group (see [BPS], [La1], [La2]).

Definition 4.1. A vector bundle $V$ on a fibered category $\mathcal{X}$ is called Nori semistable if for all smooth projective curves $C$ over an algebraically closed field and all maps $i: C \longrightarrow \mathcal{X}$ the pullback $i^{*} V$ is semistable of degree 0 .

We denote by $\operatorname{Ns}(\mathcal{X})$ the full subcategory of $\operatorname{Vect}(\mathcal{X})$ of Nori semistable vector bundles.

The S-fundamental gerbe of a fibered category $\mathcal{X}$ over $k$ is an affine gerbe $\Pi$ over $k$ together with a map $u: \mathcal{X} \longrightarrow \Pi$ whose pullback $u^{*}: \operatorname{Vect}(\Pi) \longrightarrow \operatorname{Vect}(\mathcal{X})$ is fully faithful with essential image $\operatorname{Ns}(\mathcal{X})$. The $\mathrm{S}$-fundamental gerbe is unique and is denoted by $\Pi_{\mathcal{X} / k}^{\mathrm{S}}$ when it exists.

If $\mathcal{X}$ has an S-fundamental gerbe $\Psi: \mathcal{X} \longrightarrow \Pi_{\mathcal{X} / k}^{\mathrm{S}}$ and $x \in \mathcal{X}(k)$, then the S-fundamental group $\pi^{\mathrm{S}}(\mathcal{X} / k, x)$ of $(\mathcal{X}, x)$ over $k$ is the sheaf of automorphisms of $\psi(x) \in \Pi_{\mathcal{X} / k}^{\mathrm{S}}$.

We will usually drop the $/ k$ when $k$ is clear from the context.

Remark 4.2. A fibered category $\mathcal{X}$ over $k$ has an $\mathrm{S}$-fundamental gerbe if and only if $\mathrm{H}^{0}\left(\mathcal{O}_{\mathcal{X}}\right)=k$ and $\mathrm{Ns}(\mathcal{X})$ is an abelian subcategory of $\mathrm{QCoh}(\mathcal{X})$. The "only if" is clear. For the converse observe that $\operatorname{Ns}(\mathcal{X})$ is a rigid monoidal category. If it is also an abelian subcategory of $\mathrm{QCoh}(\mathcal{X})$ then $\operatorname{Ns}(\mathcal{X})$ is $k$-Tannakian and the map $\operatorname{Ns}(\mathcal{X}) \longrightarrow \operatorname{Vect}(\mathcal{X})$ sends exact sequences to exact sequences in $\mathrm{QCoh}(\mathcal{X})$. By Tannakian duality $\operatorname{Ns}(\mathcal{X}) \simeq$ $\operatorname{Vect}(\Pi)$, where $\Pi$ is an affine gerbe, and by Remark 1.20 , the inclusion $\operatorname{Ns}(\mathcal{X}) \subseteq \operatorname{Vect}(\mathcal{X})$ is realized as the pullback of a map $\mathcal{X} \longrightarrow \Pi$, that is $\Pi=\Pi_{\mathcal{X}}^{\mathrm{S}}$.

Remark 4.3. If $\mathcal{X}$ is a fibered category with an $\mathrm{S}$-fundamental gerbe, then its profinite quotient is a Nori fundamental gerbe. In particular $\mathcal{X}$ is inflexible. Indeed since finite vector bundles on $\mathcal{X}$ are Nori semistable, we have

$$
\operatorname{EFin}(\operatorname{Vect}(\mathcal{X}))=\operatorname{EFin}(\operatorname{Ns}(\mathcal{X}))
$$

is also a $k$-Tannakian category by Theorem 1.10 and that it is an abelian subcategory of $\mathrm{QCoh}(\mathcal{X})$. From Remark 1.20 and Remark 1.21 it follows that the affine gerbe associated to $\operatorname{EFin}(\operatorname{Ns}(\mathcal{X}))$, which is the profinite quotient of $\Pi_{\mathcal{X}}^{\mathrm{S}}$, is a Nori fundamental gerbe for $\mathcal{X}$. 
Remark 4.4. Let $\phi: \mathcal{X}^{\prime} \longrightarrow \mathcal{X}$ be a map of fibered categories and $F \in \operatorname{Vect}(\mathcal{X})$. If $\mathcal{F}$ is Nori semistable then $\phi^{*} \mathcal{F}$ is Nori semistable too. The converse holds if $\phi$ is representable (by a scheme), proper and surjective. Indeed one can assume that $\mathcal{X}$ is a proper, smooth, integral curve over an algebraically closed field $k$ and must prove that $\mathcal{F}$ is semistable of degree 0. Considering a closed point in the generic fiber of $\phi$ and taking the normalization of its closure one can moreover assume that $\mathcal{X}^{\prime}$ is also a proper, smooth, integral curve over $k$. In particular $\phi$ is a cover. In this case the result follows because the pullback of a subbundle destabilizing $\mathcal{F}$ actually destabilizes $\phi^{*} \mathcal{F}$.

Example 4.5. If $X$ is a smooth, geometrically connected and geometrically projective scheme over $k$ then $X$ has an S-fundamental gerbe over $k$ (see [BPS], [La1], [La2]). Recently, it is shown in [BHD, Theorem 6.7] that if $X$ is a reduced algebraic $k$-scheme which is connected by proper chains (see [BHD, Definition 6.1]), where $k$ is algebraically closed, then $X$ has an S-fundemental gerbe over $k$.

Proposition 4.6. An affine gerbe $\Gamma$ over $k$ has an $\mathrm{S}$-fundamental gerbe over $k$.

Proof. In view of Remark 4.2 we need to show that $\mathrm{Ns}(\Gamma)$ is an abelian subcategory of $\mathrm{QCoh}(\Gamma)$. So let

$$
0 \longrightarrow K \longrightarrow F_{1} \longrightarrow F_{2} \longrightarrow Q \longrightarrow 0
$$

be an exact sequence in $\mathrm{QCoh}(\Gamma)$ with $F_{1}, F_{2} \in \mathrm{Ns}(\Gamma)$. We must show that $K, Q \in \operatorname{Ns}(\Gamma)$. Let $i: C \longrightarrow \Gamma$ be a map from a smooth projective curve over some algebraically closed field. Since $\Gamma$ is a gerbe, both $K$ and $Q$ are vector bundles. Thus $i^{*} K$ and $i^{*} Q$ are respectively kernel and cokernel, in $\mathrm{QCoh}(C)$, of a homomorphism between Nori semistable vector bundles on $C$. Since $\operatorname{Ns}(C)$ is an abelian subcategory of $\operatorname{QCoh}(C)$, it follows that $i^{*} K$ and $i^{*} Q$ are in $\operatorname{Ns}(C)$.

Lemma 4.7. Let $\mathcal{X}$ be a pseudo-proper and inflexible category over $k$, and let $f: \mathcal{Y} \longrightarrow \mathcal{X}$ be an essentially finite cover. Then

$$
\operatorname{Ns}(\mathcal{Y})=\left\{V \in \operatorname{Vect}(\mathcal{Y}) \mid f_{*} V \in \operatorname{Ns}(\mathcal{X})\right\}
$$

Proof. Given $F \in \operatorname{Vect}(\mathcal{Y})$ we have to prove that

$$
F \in \operatorname{Ns}(\mathcal{Y}) \Longleftrightarrow f_{*} F \in \operatorname{Ns}(\mathcal{X}) .
$$

Nori semistability is tested on curves. Thus we can assume that $\mathcal{X}=C$ is a smooth, integral, projective curve over an algebraically closed field $k$ : for " $\Longrightarrow$ " we know that $F \in \mathrm{Ns}(\mathcal{Y})$ and we must prove that $f_{*} F \in \mathrm{Ns}(C)$; for " $\Longleftarrow$ " we have a section $C \stackrel{i}{\longrightarrow} \mathcal{Y}$, we know that $f_{*} F \in \operatorname{Ns}(C)$ and we must prove that $i^{*} F \in \operatorname{Ns}(C)$. Here we are using the following: since $\mathcal{X}$ is pseudo-proper and inflexible, the pullback of $f_{*} \mathcal{O}_{\mathcal{X}}$ along the curve $C \longrightarrow \mathcal{X}$ is still essentially finite; see Remark 1.21.

Let $C \longrightarrow \Gamma$ be the monodromy gerbe of $f_{*} \mathcal{O}_{\mathcal{Y}}$ in EFin $(\operatorname{Vect}(C))$ and $\Delta \longrightarrow \Gamma$ the extension given in Lemma 2.2. We have $\Gamma=\mathrm{B} G$ for some finite group scheme $G$ so that the $\operatorname{map} C \longrightarrow \mathrm{B} G$ is given by a $G$-torsor $\mathcal{Q} \longrightarrow C$. Let $D$ be the normalization of an irreducible component of $\mathcal{Q}$ surjecting onto $C$. It follows that $g: D \longrightarrow C$ is a surjective cover. Since a vector bundle on $C$ is Nori semistable if and only if its pullback via $g$ is so 
(see Remark 4.4), we can replace $C$ by $D$, that is assume that $C \longrightarrow \mathrm{B} G$ factors through Spec $k$. Since the cover $\mathcal{Y} \longrightarrow C$ extends to B $G$ we know that $f: \mathcal{Y}=C \times A \longrightarrow C$ is the projection, where $A / k$ is a finite $k$-algebra. Splitting $\mathcal{Y}$ according to a decomposition of $A$ we can moreover assume $A$ local. In the case " $е$ " the inclusion $i: C \longrightarrow \mathcal{Y}=C \times A$ is induced by $A \longrightarrow k$. This map is also defined in the case " $\Longrightarrow$ " and we denote it with the same symbol $i$.

We may replace $C$ by another test curve. Hence it is enough to prove that $f_{*} F$ is semistable of degree 0 if and only if $i^{*} F$ is so. From Lemma 2.10 we obtain a sequence of surjective homomorphisms of vector bundles

$$
f_{*} F=\mathcal{G}_{N} \longrightarrow \mathcal{G}_{N-1} \longrightarrow \cdots \longrightarrow \mathcal{G}_{1} \longrightarrow \mathcal{G}_{0}=0
$$

such that $\operatorname{Ker}\left(\mathcal{G}_{l} \longrightarrow \mathcal{G}_{l-1}\right) \simeq i^{*} F$. By induction we have

$$
\operatorname{det}\left(f_{*} F\right) \simeq\left(\operatorname{det} i^{*} F\right)^{N}
$$

so that $f_{*} F$ has degree 0 if and only if $i^{*} F$ has degree 0 . Again by induction we also see that all $\mathcal{G}_{l}$ have the same slope as that of $i^{*} F$. In particular if $f_{*} F$ is semistable so is $i^{*} F \subseteq f_{*} F$. The converse is deduced from the following fact: if $0 \longrightarrow E^{\prime} \longrightarrow E \longrightarrow E^{\prime \prime} \longrightarrow 0$ is an exact sequence of vector bundles on $C$ with equal slope then $E$ is semistable if $E^{\prime}$ and $E^{\prime \prime}$ are semistable.

Proof of Theorem IV. The first claim follows from Remark 4.3. In particular by Theorem I we have 2-Cartesian diagrams

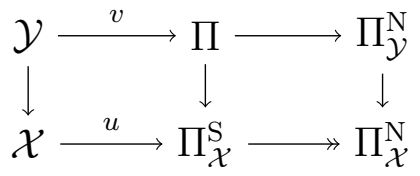

Since $\Pi_{\mathcal{X}}^{\mathrm{S}} \longrightarrow \Pi_{\mathcal{X}}^{\mathrm{N}}$ is a quotient it follows that $\Pi$ is a gerbe. $\operatorname{As} \operatorname{Ns}(\mathcal{X})$ is a full subcategory of $\operatorname{Vect}(\mathcal{X})$, by Lemma 1.22 , Lemma 2.7 and Lemma 4.7 we have that $\operatorname{Vect}(\Pi) \longrightarrow \operatorname{Vect}(\mathcal{Y})$ is fully faithful with essential image $\operatorname{Ns}(\mathcal{Y})$. Thus we have $\Pi=\Pi_{\mathcal{Y}}^{\mathrm{S}}$. The last claim follows from the Cartesian diagram and Lemma 1.17.

\section{Counterexamples}

In this section we collect various examples. We start by showing that, under the assumption of Theorem I, the condition $\mathrm{H}^{0}\left(\mathcal{O}_{\mathcal{Y}}\right)=k$ in general does not imply that $\mathcal{Y}$ is inflexible, even when $f_{*} \mathcal{O}_{\mathcal{Y}}$ has étale monodromy gerbe (e.g. if char $\left.k=0\right)$.

Example 5.1. Let $k$ be an algebraically closed field. We show an example of an elliptic curve $X$ over $k$ with an essentially finite cover $f: Y \longrightarrow X$ of degree 2 such that $\mathrm{H}^{0}\left(\mathcal{O}_{\mathcal{Y}}\right)=k$ but $Y$ is not inflexible. Clearly here $Y$ is not reduced. The monodromy gerbe of $f_{*} \mathcal{O}_{Y}$ is $\mathrm{B} \mu_{2}$.

Let $X$ be an elliptic curve together with a non trivial line bundle $\mathcal{L}$ such that $\mathcal{L}^{\otimes 2} \simeq \mathcal{O}_{X}$. This is the data of a Nori-reduced map $u: X \longrightarrow \mathrm{B} \mu_{2}$. Let $A=k[\epsilon]=k[x] /\left(x^{2}\right)$ equipped 
with the $\mu_{2}=\operatorname{Spec}\left(k[y] /\left(y^{2}-1\right)\right)$ action

$$
A \longrightarrow A \otimes\left(k[y] /\left(y^{2}-1\right)\right), \epsilon \longmapsto \epsilon \otimes y
$$

and set $\Delta=\left[\operatorname{Spec} A / \mu_{2}\right]$. Define $Y \longrightarrow X$ with the 2-Cartesian diagram

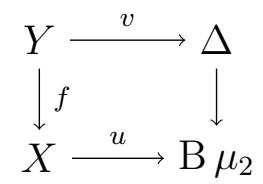

Since $u$ is Nori-reduced we have $u_{*} \mathcal{O}_{X} \simeq \mathcal{O}_{\mathrm{B} \mu_{2}}$ and $v_{*} \mathcal{O}_{Y} \simeq \mathcal{O}_{\Delta}$ by flat base change. In particular

$$
\mathrm{H}^{0}\left(\mathcal{O}_{Y}\right)=\mathrm{H}^{0}\left(\mathcal{O}_{\Delta}\right)=A^{\mu_{2}}=k
$$

where the last equality follows from a direct computation. On the other hand, $Y$ is not inflexible because $v_{*} \mathcal{O}_{Y} \simeq \mathcal{O}_{\Delta}$; this implies that $v: Y \longrightarrow \Delta$ does not factor through a gerbe.

We now show examples of towers without a Galois envelope. The lemma below will be our method to exclude that a given tower has a Galois envelope.

A torsor under a finite group scheme $G$ over $k$ is called minimal if it does not come from a torsor under a proper subgroup of $G$. For instance Nori-reduced torsors are minimal.

Lemma 5.2. Let $\mathcal{X}$ be a fibered category, and let $\mathcal{Z} \longrightarrow \mathcal{Y} \longrightarrow \mathcal{X}$ be a $(G, H)$-tower of torsors with $\mathcal{Y} \longrightarrow \mathcal{X}$ minimal. If the tower has a Galois envelope with group $\mathcal{G}$ then the map $\mathcal{X} \longrightarrow \mathrm{B}(G, H)$ factors through a map $\mathrm{B} \mathcal{G} \longrightarrow \mathrm{B}(G, H)$.

Proof. Let $\mathcal{P} \longrightarrow \mathcal{X}$ and $\mathcal{P} \longrightarrow \mathcal{Z}$ be the Galois envelope. We must show that there is a tower over $\mathrm{B} \mathcal{G}$ whose pullback along $\mathcal{X} \longrightarrow \mathrm{B} \mathcal{G}$ is the original tower. Since $\mathcal{Y} \longrightarrow \mathcal{X}$ is minimal the map $\mathcal{G} \longrightarrow G$ is surjective. By Lemma 1.17 the $G$-torsor over B $\mathcal{G}$ induced by $\mathcal{G} \longrightarrow G$ is $\mathrm{B} \mathcal{H} \longrightarrow \mathrm{B} \mathcal{G}$, where $\mathcal{H}$ is the kernel of $\mathcal{G} \longrightarrow G$. The pullback of the $G$-torsor $\mathrm{B} \mathcal{H} \longrightarrow \mathrm{B} \mathcal{G}$ along $\mathcal{X} \longrightarrow \mathrm{B} \mathcal{G}$ is, by construction, $\mathcal{Y} \longrightarrow \mathcal{X}$. The map $\mathcal{Y} \longrightarrow \mathrm{B} \mathcal{H}$ is given by the $\mathcal{H}$-torsor $\mathcal{P} \longrightarrow \mathcal{Y}$. The homomorphism $\mathcal{H} \longrightarrow H$ gives a map $\mathrm{B} \mathcal{H} \longrightarrow \mathrm{B} H$ and therefore an $H$-torsor $\mathcal{B} \longrightarrow \mathrm{B} \mathcal{H}$. The fact that $\mathcal{P} \longrightarrow \mathcal{Z}$ is $\mathcal{H}$-equivariant means that $\mathcal{Z} \longrightarrow \mathcal{Y}$ is the $H$-torsor induced by the $\mathcal{H}$-torsor $\mathcal{P} \longrightarrow \mathcal{Y}$ along $\mathcal{H} \longrightarrow H$. This exactly means that the $H$-torsor $\mathcal{B} \longrightarrow \mathrm{B} \mathcal{H}$ pulls back to $\mathcal{Z} \longrightarrow \mathcal{Y}$ along $\mathcal{Y} \longrightarrow \mathrm{B} \mathcal{H}$.

This example shows that the condition on the cohomology groups $\mathrm{H}^{1}$ in Theorem III is necessary.

Example 5.3. Assume that $G$ and $H$ are not étale. We give an example of a pseudoproper, inflexible and smooth algebraic stacks $\mathcal{X}$ with a pointed $(G, H)$-tower of Norireduced torsors $\mathcal{Z} \longrightarrow \mathcal{Y} \longrightarrow \mathcal{X}$ without a Galois envelope. In particular $\mathcal{Z} \longrightarrow \mathcal{X}$ cannot be an essentially finite cover by Theorem 3.10 .

Using notations of Lemma 3.6 set $\mathcal{X}=\mathrm{B} Q$ with the tower $\mathrm{B} W^{\prime} \longrightarrow \mathrm{B} W_{G}(H) \longrightarrow \mathrm{B} Q$. If this tower has a Galois envelope then by Lemma 5.2 the map $\mathrm{B} Q \longrightarrow \mathrm{B}(G, H)$ factors through a finite gerbe. Since the map B $Q \longrightarrow \mathrm{B}(G, H)$ is fully faithful, this means that $Q$ 
has to be a finite group scheme. Thus we must show that $Q$ is not a finite group scheme. In particular we can assume $k$ to be algebraically closed, so that $G$ is a disjoint union of copies

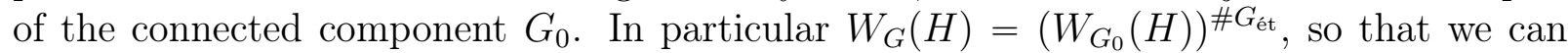
assume $G$ local but not trivial. Moreover there is an injective map $W_{G}\left(H_{0}\right) \longrightarrow W_{G}(H)$, where $H_{0}$ is the connected component of $H$. Thus we can also assume that $H$ is local but not trivial. If $k[\epsilon]=k[x] /\left(x^{2}\right)$ there is a map $k[\epsilon] \subseteq k[G]$. Thus one gets an injective map of group schemes $W_{U}(H) \longrightarrow W_{G}(H)$ where $U=\operatorname{Spec} k[\epsilon]$. Similarly one can find a closed embedding $U \longrightarrow H$, which yields a monomorphism of schemes $W_{U}(U) \longrightarrow W_{U}(H)$. Moreover there is a monomorphism

$$
\mathbb{A}^{1}(B) \longrightarrow W_{U}(U)(B)=\operatorname{Hom}_{B \text {-algebras }}(B[\epsilon], B[\epsilon]), b \longmapsto(\epsilon \mapsto b \epsilon)
$$

In conclusion we find a monomorphism $\phi: \mathbb{A}^{1} \longrightarrow W_{G}(H)$. If $W_{G}(H)$ is finite, the image of $\phi$ must be connected, reduced, finite and with a rational point, that is $\operatorname{Spec} k$, so that $\phi$ is not a monomorphism. Therefore, $W_{G}(H)$ is not finite.

The next example shows the importance of the pseudo-properness assumption on $\mathcal{X}$ in Theorem III.

Example 5.4. We give an example of a smooth, integral and affine scheme $X$ over $k$ with a pointed $(G, H)$-tower of Nori-reduced torsors without a Galois envelope.

Assume that $k$ is an algebraically closed field of characteristic 2 and let $H=\mu_{2}$ and $G$ be either $\mu_{2}$ or $\alpha_{2}$. Recall that if $B$ is a $k$-algebra and $b \in B^{*}$ then $B[x] /\left(x^{2}-b\right)$ has an action by $\mu_{2}$ and an action by $\alpha_{2}$ and it is a torsor over $B$ for both actions. Since $k$ is algebraically closed and $\alpha_{2}$ and $\mu_{2}$ are simple we have that $B[x] /\left(x^{2}-b\right)$ is Nori-reduced if $b$ is not a square in $B$ and it is trivial otherwise.

Let $K$ be the separable closure of the field of fractions $k(t)$ and consider

$$
K_{1}=K[x] /\left(x^{2}-t\right) \text { and } K_{2}=K[x, y] /\left(x^{2}-t, y^{2}-x\right)
$$

The rings $K_{1} \subseteq K_{2}$ are fields. Thus $\operatorname{Spec} K_{2} \longrightarrow \operatorname{Spec} K_{1} \longrightarrow \operatorname{Spec} K$ is a $(G, H)$-tower of non trivial torsors which defines a map $\xi: \operatorname{Spec} K \longrightarrow \mathrm{B}(G, H)$. Consider a smooth map $X \longrightarrow \mathrm{B}(G, H)$ from a connected affine scheme and whose image contains the point $\xi$. We claim that the corresponding tower is pointed and Nori-reduced. It is pointed because $k$ is algebraically closed. It is Nori-reduced because, since $K$ is separably closed, the map $\xi$ factors through a map Spec $K \longrightarrow X$ and the torsors in the tower $\xi$ are not trivial.

Let $\alpha: X \longrightarrow \mathrm{B}(G, H)$ and $\beta: Y \longrightarrow \mathrm{B}(G, H)$ be smooth maps from connected affine schemes and assume that their images contain $\xi$ and the trivial tower respectively. If the tower $\alpha$ does not have a Galois envelope we have our counter-example. Otherwise, by 5.2 , the map is (topologically) constant and $\xi$ is an open point in $\mathrm{B}(G, H)$. If $\mathrm{B}(G, H)$ is irreducible then $\xi$ would be its generic point and, since the image of $\beta$ is open, it would contain $\xi$. In this case if the tower $\beta$ has a Galois envelope it would follow that $\xi$ is the trivial tower, that is $x \in K_{1}$ become a square extending the field $K$, which is not true.

Thus it is enough to show that $\mathrm{B}(G, H)$ is a smooth and connected algebraic stack. Consider the tower $\mathcal{W} \longrightarrow \underline{\operatorname{Hom}}_{k}(G, \mathrm{~B} H) \longrightarrow \mathrm{B}(G, H)$ described in 3.2. It is enough to show that $\mathcal{W}$ is a smooth connected algebraic stack. The objects of $\mathcal{W}(B)$ are $H$-torsors 
over $G \times B$ with a trivialization over $\operatorname{Spec} B \stackrel{1}{\longrightarrow} G \times B$. We think about $\mu_{2}$-torsors as line bundles with an isomorphism between its square and the trivial bundle. Set also $B[\epsilon]=B[x] /\left(x^{2}\right)=B[G]$. Thus $\mathcal{W}(B)$ is the stack of triples $(L, \phi, \psi)$ where $L$ is a $B[\epsilon]$ line bundle, $\phi: L^{2} \longrightarrow B[\epsilon]$ is an isomorphism and $\psi: L / \epsilon L \longrightarrow B$ is an isomorphism such that $\psi^{\otimes 2} \equiv \phi$ modulo $\epsilon$. If $L=B[\epsilon]$ then $\phi=a+b \epsilon \in B[\epsilon]^{*}$ and $\psi=c \in B^{*}$ with $a=c^{2}$ and, up to an isomorphism in $\mathcal{W}(B)$, one can always assume $c=1$. Thus the map $\mathbb{A}^{1} \longrightarrow \mathcal{W}$, mapping $b \in \mathbb{A}^{1}(B)$ to $(B[\epsilon], 1+b \epsilon, 1)$, is an epimorphism in the Zariski topology. In particular $\mathbb{A}^{1} \longrightarrow \mathcal{W}$ is an fppf covering if $\mathbb{A}^{1} \times \mathcal{W} \mathbb{A}^{1} \longrightarrow \mathbb{A}^{1}$ is. A direct computation shows that an isomorphism $(B[\epsilon], 1+b \epsilon, 1) \longrightarrow(B[\epsilon], 1+c \epsilon, 1)$ exists if and only if $b=c$ and in this case it is the multiplication by $1+\lambda \epsilon$ for $\lambda \in B$. This means that $\mathbb{A}^{1} \times_{\mathcal{W}} \mathbb{A}^{1} \longrightarrow \mathbb{A}^{1}$ coincides with the projection $\mathrm{pr}_{1}: \mathbb{A}^{1} \times{ }_{k} \mathbb{A}^{1} \longrightarrow \mathbb{A}^{1}$ which is an fppf covering.

We conclude the section with an example showing that Corollary I (and thus Theorem I) as well as Lemma 2.11 fails without the finiteness assumption on the first cohomology group of vector bundles.

Example 5.5. We give an example of a Nori-reduced torsor $f: \mathcal{Y} \longrightarrow \mathcal{X}$ between pseudoproper, inflexible and smooth algebraic stacks over $k$ with $y \in \mathcal{Y}(k)$ such that the following hold:

- $f_{*}$ does not map essentially finite vector bundles to essentially finite vector bundles,

- $\pi^{\mathrm{N}}(\mathcal{X}, f(x))$ is finite,

- $f: \mathcal{Y} \longrightarrow \mathcal{X}$ is the universal torsor, but

- $\pi^{\mathrm{N}}(\mathcal{Y}, y)$ is not trivial.

Let $k$ be a field of characteristic $2, H=\mu_{2}$, and let $G$ be either $\mu_{2}$ or $\alpha_{2}$. Consider the $(G, H)$-tower of pointed Nori-reduced torsors

$$
\mathcal{Z}=\mathrm{B} W^{\prime} \stackrel{h}{\longrightarrow} \mathcal{Y}=\mathrm{B} W_{G}\left(\mu_{2}\right) \stackrel{f}{\longrightarrow} \mathcal{X}=\mathrm{B} Q
$$

introduced in Example 5.3. Since $\mathcal{Z} \longrightarrow \mathcal{X}$ is not essentially finite it follows that $h_{*} \mathcal{O}_{\mathcal{Z}}$ is essentially finite while $f_{*}\left(h_{*} \mathcal{O}_{\mathcal{Z}}\right)$ is not.

Consider $y$ and $x=f(y)$ as the trivial torsors. Since the Nori fundamental group of an affine gerbe B $S$ pointed at the trivial torsor is the profinite quotient $\widehat{S}$ of $S$, we must show that $\widehat{Q}=G$ and $\widehat{W_{G}\left(\mu_{2}\right)}=\mu_{2}$. Given a $k$-algebra $B$ set $B[\epsilon]=B[x] /\left(x^{2}\right)$. We have

$$
W_{G}\left(\mu_{2}\right)(B)=\mu_{2}(B[\epsilon])=\left\{a+b \epsilon \mid a^{2}=1\right\}
$$

from which it is easy to conclude that $W_{G}\left(\mu_{2}\right)=\mathbb{G}_{a} \times \mu_{2}$. Since any homomorphism from $\mathbb{G}_{a}$ to a profinite group is trivial we conclude that $\widehat{W_{G}\left(\mu_{2}\right)}=\mu_{2}$. Similarly, denoting by $K$ the kernel of $Q \longrightarrow \widehat{Q}$, we have $\mathbb{G}_{a} \subseteq K \subseteq W_{G}\left(\mu_{2}\right)$. Since $\mu_{2}$ is simple we just have to check that $\mathbb{G}_{a}$ is not normal in $Q$. Note that $G=$ Spec $k[\epsilon]$; for $g \in G(B)$, let $\psi_{g}: B[\epsilon] \longrightarrow B[\epsilon]$ be the multiplication by $g$. Then,

$$
g \star x \star g^{-1}=\psi_{g}(x) \text { for } x \in \mu_{2}(B[\epsilon])=W_{G}\left(\mu_{2}\right)(B)
$$


where $\star$ denotes the multiplication in $Q$. If $G=\mu_{2}$, so that $g \in B^{*}$ with $g^{2}=1$, an easy computation shows that $\psi_{g}(\epsilon)=g \epsilon+(g-1)$. Thus

$$
\psi_{g}(1+\epsilon)=g+g \epsilon
$$

is in $\mathbb{G}_{a}$ if and only if $g=1$, which is not always the case.

If $G=\alpha_{2}$, so that $g \in B$ with $g^{2}=0$, then $\psi_{g}(\epsilon)=\epsilon+g$. Thus

$$
\psi_{g}(1+\epsilon)=1+g+\epsilon
$$

is in $\mathbb{G}_{a}$ if and only if $g=0$, which is not always the case.

\section{REFERENCES}

[ABE] M. Antei, I. Biswas and M. Emsalem, Towers of torsors over a field, arXiv:1606.08671 (will not be published).

[AM] M. Antei and V. B. Metha, Vector Bundles over Normal Varieties Trivialized by Finite Morphisms, Archiv Math. 97 (2011), 523-527.

[BdS] I. Biswas and J. P. P. dos Santos, Vector bundles trivialized by proper morphisms and the fundamental group scheme, Jour. Inst. Math. Jussieu 10 (2011), 225-234.

[BHD] I. Biswas, P. H. Hai and J. P. dos Santos, On the Fundamental Group Schemes of Certain Quotient Varieties, https://arxiv.org/pdf/1809.06755.pdf, 2018.

[BPS] I. Biswas, A. J. Parameswaran and S. Subramanian, Monodromy group for a strongly semistable principal bundle over a curve, Duke Math. Jour. 132 (2006), 1-48.

[BV] N. Borne and A. Vistoli, The Nori fundamental gerbe of a fibered category, J. Algebraic Geom. 24 (2015), 311-353.

[De] P. Deligne, Catégories Tannakiannes, The Grothendieck Festschrift, Vol. II, Progr. Math., vol. 87, Birkhäuser Boston, Boston, MA, pp. 111-195, 1990.

[DG] M. Demazure, P. Gabriel, Groupes Algébriques, Tome I, MASSON \& CIE, Éditeur - Paris, 1970.

[EHS] H. Esnault, P. H. Hai and X. Sun, On Nori's fundamental group scheme, Progress in Mathematics 265, 377-398, Birkhäuser, 2007.

[Fal] G. Faltings, Finiteness of coherent cohomology for proper fppf stacks, J. Algebraic Geom. 12 (2003), 357-366.

[Ga] M. Garuti, On the "Galois closure" for torsors, Proc. Am. Math. Soc. 137 (2009), 3575-3583.

[HR] J. Hall and D. Rydh, The Hilbert stack, Adv. Math. 253 (2014), 194-233.

[La1] A. Langer, On the $S$-fundamental group scheme, Ann. Inst. Fourier 61 (2011), 2077-2119.

[La2] A. Langer, On the $S$-fundamental group scheme II, Jour. Inst. Math. Jussieu 11 (2012), 835-854.

[No1] M. Nori, The fundamental group schemes, Proc. Indian Aacd. Sci. (Math. Sci.) 91 (1982), $73-122$.

[No2] M. Nori, The fundamental group-scheme of an abelian variety, Math. Ann. 263 (1983), 263-266.

[Otabe] S. Otabe, Lifting problem for linearly reductive torsors of curves, arXiv: https://arxiv.org/abs/1802.01111, 2018.

[Sz] T. Szamuely, Galois Groups and Fundamental Groups, Cambridge Studies in Advanced Mathematics, Vol. 117, 2009.

[Ton] F. Tonini, Sheafification functors and Tannaka's reconstruction, https://arxiv.org/pdf/1409.4073.pdf, 2014.

[TZ1] F. Tonini and L. Zhang, Algebraic and Nori Fundamental Gerbe, Journal of the Institute of Mathematics of Jussieu, 1-43. doi:10.1017/S147474801700024X, 2017.

[TZ2] F. Tonini and L. Zhang, F-divided sheaves trivialized by dominant maps are essentially finite, to appear in Trans Am Math Soc., 2018. 
[TZ3] F. Tonini and L. Zhang, Essentially Finite Vector Bundles on Normal Pseudo-proper Algebraic Stacks, arXiv:1702.03751 (2017), 9.

[Wat] W. C. Waterhouse, Introduction to affine group schemes, Springer-Verlag, New York, 1979.

[Zh] L. Zhang, Nori's fundamental group over a non algebraically closed field, Ann. Sc. Norm. Super. Pisa Cl. Sci. (5) Vol. XVIII, 1349-1394, 2018.

Universidad de Costa Rica, Ciudad universitaria Rodrigo Facio Brenes, Costa Rica

E-mail address: marco.antei@ucr.ac.cr

School of Mathematics, Tata institute of Fundamental Research, Homi Bhabha Road, BOMBAY 400005, INDIA

E-mail address: indranil@math.tifr.res.in

Laboratoire Paul Painlevé, U.F.R. de Mathématiques, Université des Sciences et des Technologies de Lille 1, 59655 Villeneuve D'AscQ, France

E-mail address: emsalem@math.univ-lille1.fr

Universitá degli Studi di Firenze, Dipartimento di Matematica e Informatica 'Ulisse Dini', Viale Morgagni, 67/A, Firenze, 50134 Italy

E-mail address: fabio.tonini@unifi.it

The Chinese University of Hong Kong, Department of Mathematics, Shatin, New TerRITORIES, HONG KONG

E-mail address: lzhang@math.cuhk.edu.hk 